(2) Open Access Full Text Article

REVIEW

\title{
Immunotherapy For Ovarian Cancer: Recent Advances And Combination Therapeutic Approaches
}

This article was published in the following Dove Press journal: OncoTargets and Therapy

\author{
Innocenza Palaia (D* \\ Federica Tomao (1)* \\ Carolina Maria Sassu \\ Lucia Musacchio (iD) \\ Pierluigi Benedetti Panici \\ Department of Maternal and Child \\ Health and Urological Sciences, \\ "Sapienza" University of Rome, \\ Policlinico Umberto I, Rome 0016I, Italy \\ *These authors contributed equally to \\ this work
}

Correspondence: Innocenza Palaia Department of Maternal and Child Health and Urological Sciences, "Sapienza"

University of Rome, Policlinico Umberto

I, Viale Del Policlinico 155, Rome 0016I, Italy

Tel +39064940550

Email innocenza.palaia@uniromal.it

\begin{abstract}
Epithelial ovarian cancer (EOC) is the most lethal gynaecological cancer. Although many advances have been made in therapeutic strategies, the global standard of care still remains radical surgery plus chemotherapy, but new scenarios need to be explored to improve survival. The role of immunotherapy in EOC treatment is controversial. Results obtained from studies evaluating immunotherapy are contradictory: in particular data on survival are not as good as expected when immunotherapy was administered alone, and other data are still immature. Thus, significant efforts must be devoted to finding new strategies for the use of immunotherapy. The aim of this paper is to review the most recent findings of the use of immunotherapy in ovarian cancer, with a particular focus on combination approaches. Keywords: immunotherapy, ovarian cancer, adoptive cell therapy, combination strategies, therapeutic vaccination, immune-checkpoint inhibitors
\end{abstract}

\section{Introduction}

Epithelial ovarian cancer (EOC) is the most lethal gynecological cancer in developed countries with 22,530 estimated new cases and 13,980 deaths in 2019 in the USA. ${ }^{1}$ Although many advances have been made in therapeutic strategies, the global standard of care for the past 20 years has been radical surgery and platinum/taxane-based chemotherapy eventually associated with bevacizumab. ${ }^{2,3}$ Currently, the initial response rate (RR) is $60-80 \%$; nonetheless, $70 \%$ of advancedstage patients will relapse within 5 years, and many of them develop drug-resistant disease. $^{4}$ For platinum-resistant (Platinum Free Interval (PFI) $>1$ and $<6$ months) or refractory (disease progression during the last line of platinum therapy or within 4 weeks from the last platinum dose) patients the life expectancy does not exceed 1 year; indeed, most patients sensitive to first-line therapy also experience a relapse within 2 years from diagnosis, so it is essential to find new attack strategies to improve survival. The advent of poly (ADP-ribose) polymerase (PARP) inhibitors (PARPis) has certainly significantly improved the outcomes of EOC patients, both for Breast Related Cancer Antigens (BRCA)-mutant and for BRCA-wild type tumors, but new therapy scenarios need to be explored. ${ }^{5}$

Immunotherapy, which recognizes different approaches, had a strong growth in recent years and experienced encouraging results in melanoma, non-small cell lung cancer, kidney and urothelial cancers. ${ }^{6,7}$ EOC has long been considered a poorly immunogenic neoplasm, but evidence of mechanisms of immune evasion, 
spontaneous tumor regressions ${ }^{8,9}$ and responses to immunecheckpoint inhibitors (ICIs) have proven otherwise. ${ }^{10}$ Many studies have been conducted, and some evidences show that EOC could benefit from immunotherapy.

Immunotherapy in Ovarian Cancer: Background

Immunotherapy knows three different strategies (Table 1): active immunotherapy, passive immunotherapy and immunomodulation. The first has the aim of stimulating an antitumor response from the patient's own immune system itself inducing also an immunological memory. Passive immunotherapy uses the administration of immune components that directly act and promote an anti-tumor response. Finally, "immunomodulation" includes all those approaches, hardly classifiable, that enhance general immune responsiveness. ${ }^{11}$

The potential role of the immune response has been investigated in EOC and in some studies a correlation was found between the presence of tumor-infiltrating lymphocytes (TILs), the expression of PD-1 and the overall survival (OS). ${ }^{12,13}$

According to the status of TILs, tumors can be classified into two categories. "Inflamed tumors" are characterized by the presence of a high density of $\mathrm{CD}^{+} \mathrm{T}$ cells, so could benefit from therapies acting on T cell checkpoint implicated in immune-tolerance. In the "non-inflamed tumors" $\mathrm{T}$ cells are absent in tumor beds and tumor edges. So, this type of tumors is generally affected by a failure in $\mathrm{T}$ cell priming and needs strategies that could deliver autologous/allogenic effector cells into the cancer. ${ }^{14,15}$

Other types of tumors, defined as "immune-excluded", are characterized by the modification of tumor microenvironment (TME) and the presence of inhibitory cells that prevent $\mathrm{CD}^{+} \mathrm{T}$ cells from entering the tumor islets, even if they are present in the stroma. Such patients could benefit from strategies aiming to increase the infiltrations of tumors by immune effector cells such as T cell tracking modulators, epigenetic modulators, TME remodeling molecules, radiation therapy. ${ }^{16}$

BRCA 1/2 mutated high grade serous ovarian cancer (HGSOC) shows a higher mutational load and increased expression of TILs, programmed cell death (PD-1) and its ligand (PD-L1). Furthermore, patients with T-cell-rich tumors experience longer progression-free and OS, ${ }^{12}$ while immune evasion mechanisms are associated with poor survival. ${ }^{17,18}$

The aim of this paper is to review the most recent data and the ongoing studies on immunotherapy in ovarian cancer.

A search in Pubmed was performed combining the following keywords: "immunotherapy", “immune environment", "ovarian cancer", "PD1/PD-L1 expression", to retrieve preclinical data. A search in PubMed with keywords "ovarian cancer", "immune-checkpoint inhibitors",

Table I Types of Cancer Immunotherapies

\begin{tabular}{|c|c|c|c|c|}
\hline Mechanism of Action & \multicolumn{4}{|l|}{ Classes } \\
\hline \multirow[t]{2}{*}{ Active } & $\begin{array}{l}\text { Cancer vaccines } \\
\text { (preventive/treatment) }\end{array}$ & \multicolumn{3}{|l|}{$\begin{array}{l}\text { Dendritic cells } \\
\text { Peptide } \\
\text { Allogenic }\end{array}$} \\
\hline & Immune-checkpoint inhibitors & \multicolumn{3}{|l|}{$\begin{array}{l}\text { Anti-CTLA-4 } \\
\text { Anti-PD- I/PD-LI }\end{array}$} \\
\hline \multirow[t]{4}{*}{ Passive } & \multicolumn{4}{|l|}{ Monoclonal antibodies (MABs) } \\
\hline & \multicolumn{4}{|l|}{ Cytokines } \\
\hline & \multirow[t]{2}{*}{ Adoptive cell transfer } & MHC-independent & Genetically modified & $\begin{array}{l}\text { NK cells } \\
\text { LAK cells } \\
\text { CIK cells } \\
\text { CAR cells }\end{array}$ \\
\hline & & MHC-dependent & Genetically modified & $\begin{array}{l}\text { TIL cells } \\
\text { TCR cells }\end{array}$ \\
\hline Immunomodulation & Drugs hardly classifiable & \multicolumn{3}{|l|}{$\begin{array}{l}\text { IDO inhibitors } \\
\text { COX-2 inhibitors }\end{array}$} \\
\hline
\end{tabular}

Abbreviations: CAR, chimeric antigen receptor; CIK, cytokine-induced killer cells; COX-2, cyclooxygenase 2; CTLA-4, cytotoxic T lymphocyte-associated protein 4; IDO, indoleamine 2,3-dioxygenase; LAK, lymphokine activated killer; MHC, major histocompatibility complex; NK, natural killer; PD-I, programmed death I; PD-LI, programmed death ligand I; TCR, T cell receptor; TIL, gtumor-infiltrating lymphocytes. 
“adoptive cell therapy" and "vaccination" was performed to retrieve published clinical trials. ClinicalTrial.gov was searched for ongoing trials with the same keywords.

\section{Mono-Immunotherapy in Ovarian Cancer}

In the last few years, some studies have been published on immunotherapy in EOC mainly involving ICIs, that block immune-checkpoint such as cytotoxic $\mathrm{T}$ lymphocyteassociated protein 4 (CTLA-4) or the programmed death 1 (PD-1) receptor, alone or in combination with other drugs. Hodi et al first showed antitumor effects of ipilimumab (anti-CTLA-44) in nine patients with stage IV ovarian cancer (OC) patients, after vaccination with irradiated, autologous tumor cells engineered to produce Granulocyte-Macrophage Colony-Stimulating Factor (GVAX). In one patient, an objective radiographic response was noted and multiple infusions of anti-CTLA -4 antibody every 3 to 5 months have maintained disease control over 4 years; also, three patients showed disease stability lasting 6, 4 and 2 months, respectively,y as confirmed by $\mathrm{Ca} 125$ (Cancer Antigen 125) levels and radiological evaluation, without important toxicities. ${ }^{19,20}$ In 2015, our group reviewed data on ICIs as monotherapy in recurrent platinum-sensitive (PFI $>12$ months) or platinum-resistant EOC in second-line, third-line, or fourthline, concluding that the results can surely be promising in the next future. ${ }^{21}$ However, even if the biology of the tumor suggested that EOC patients could potentially benefit from immunotherapy, up to now the results obtained from mono-immunotherapy are not proved to be as satisfactory as in other neoplasms. For example, the use of single-agent antibodies targeting the CTLA-4 or PD-1 or PD-L1 showed modest results in EOC with median RRs of 10-15\%. Moreover, a control of disease was showed only in less than half of enrolled women. ${ }^{2-25}$ In 2013, Sabbatini et al published data from Phase III clinical trial to evaluate whether abagovomab (Anti-Human CA-125 antibody) maintenance therapy prolongs recurrence-free survival (RFS) and OS in patients with ovarian cancer in first clinical remission. Authors found that abagovomab administered as maintenance therapy for advanced ovarian cancer patients in the first remission after debulking surgery and platinum-based chemotherapy does not prolong RFS or OS. ${ }^{26}$ In addition, a subanalysis of the MIMOSA trial was conducted to evaluate whether abagovomab induces protective immune responses in ovarian cancer patients in first clinical remission. Authors measured circulating CA125-specific cytotoxic T lymphocytes (CTL) and human antimouse antibody and anti-anti-idiotypic (Ab3) before starting the treatment and at different time points during induction and maintenance phases showing that abagovomab does not induce CA125-specific CTL. However, patients with CA125-specific CTL perform better than patients without, irrespective of abagovomab treatment. Abagovomab-induced Ab3 is associate with prolonged RFS in patients without CA125-specific CTL. $^{27}$ In light of these observations, Battaglia et al evaluated whether the assessment of the immune system status before of abagovomab administration might predict sensitivity to it. Authors found that patients on abagovomab with IFN- $\gamma$ producing $\mathrm{CD}^{+} \mathrm{T}$ cell percentage and absolute count above the cutoff had a better RFS $(\mathrm{P}=0.042$ and $\mathrm{P}=0.019$, respectively), compared to patients with IFN- $\gamma$ producing $\mathrm{CD}^{+} \mathrm{T}$ cell percentage and absolute count below the cutoff. ${ }^{28}$

Considering these controversial data, in the last few years more attention has been paid to new strategies with a special focus on the combination therapy and unconventional immunotherapeutic agents (Table 2), which could potentiate the positive effects of immunotherapy.

\section{Combination Strategies} Immunotherapy in Combination with Anti Angiogenic Agents

Anti-VEGF (Vascular Endothelial Growth Factor) therapy may enhance response to immunotherapy. In a murine syngenetic model of EOC overexpressing VEGF, VEGF

Table 2 Strategies to Enhance the Efficacy of Immunotherapy

\begin{tabular}{|l|l|}
\hline \multicolumn{2}{|l|}{ Combinations Strategies } \\
\hline $\begin{array}{l}\text { IMMUNE-CHECKPOINT } \\
\text { INHIBITORS }\end{array}$ & $\begin{array}{l}\text { ANTI ANGIOGENIC AGENTS } \\
\text { Plus } \\
\text { CHEMOTHERAPY } \\
\text { OTHER IMMUNE-CHECKPOINT } \\
\text { INHIBITORS } \\
\text { OTHER AGENTS }\end{array}$ \\
\hline Other Strategies & $\begin{array}{l}\text { MONOTHERAPY } \\
\text { COMBINATION }\end{array}$ \\
\hline ACT & $\begin{array}{l}\text { MONOTHERAPY } \\
\text { COMBINATION }\end{array}$ \\
\hline VACCINE
\end{tabular}

Abbreviations: ACT, adoptive cell therapy; PARPis, PARP inhibitors. 
plus $\mathrm{PGE}_{2}$ blockade increased T-cell homing to tumors, ${ }^{29}$ whereas, in a murine melanoma model, disruption of the VEGF/VEGF receptor 2 axis was shown to increase extravasation of adoptively transferred $\mathrm{T}$ cells into the tumor and to improve adoptive cell transfer immunotherapy. ${ }^{30}$ In addition, anti-VEGF strategies in combination with a granulocyte-macrophage colony-stimulating factorsecreting tumor vaccine have been shown to reduce the number of $\mathrm{CD} 4^{+} \mathrm{CD} 25^{+}$Tregs, which leads to increased CTL induction and improved vaccine efficacy. ${ }^{31}$ In a Phase I study, the combination of durvalumab (anti-PDL1) and cediranib (an oral VEGF receptor inhibitor) was tested in 14 recurrent or metastatic ovarian cancers even with more than 5 previous chemotherapy lines. The disease control rate was $75 \%$ with $50 \%$ RRs (6 Partial Response PR and 3 stable disease SD) assessed by imaging using response evaluation criteria in solid tumors (RECIST) v1.1 criteria. Recurrent grade 2 and non-doselimiting toxicity grade 3 and 4 adverse events (AEs) have occurred on the daily schedule (hypertension $2 / 8$ patients, diarrhea $2 / 8$ pulmonary embolism $2 / 8$, pulmonary hypertension $1 / 8$ and lymphopenia $1 / 8$ ) while in durvalumab plus intermittent cediranib schedule grade 3 and 4 AEs were hypertension $1 / 6$ and fatigue 1/6. Authors concluded that durvalumab plus intermittent cediranib is a tolerable and clinically active combination in this setting of patients. $^{32}$ Most recently, a Phase II trial using combined nivolumab (anti-PD-1) and bevacizumab (anti-VEGF monoclonal antibody) showed clinical activity in 38 women affected by recurrent ovarian cancer after 1 to 3 lines of prior chemotherapy. The Objective Response Rate (ORR) was 28.9\% (95\% exact binomial CI, 15.4-45.9\%) with better results in platinum-sensitive setting (ORR 40\%, [CI 19.1-64.0\%] vs 16.7\%, [CI 3.6-41.4\%] in platinum-resistant disease). Median Progression Free Survival (PFS) by RECIST was 9.4 months (95\% CI, 6.7 months to not available (NA)): 12.1 months (95\% CI, 8.4 months to NA) in platinum-sensitive patients vs 7.7 months $(95 \% \mathrm{CI}$, 4.7 months to NA) in platinum-resistant ones. Thirty-four participants $(89.5 \%)$ experienced at least 1 treatmentrelated adverse event and $23.7 \%$ a grade 3 or higher treatment-related adverse event. ${ }^{33}$

\section{Immunotherapy in Combination with PARPis}

PARPis can activate interferon signaling and activate stimulator of interferon genes in BRCA-deficient tumors and synergize with PD-1 or CTLA-4 blockade in mouse models. $^{34-36}$

Of note, PARPis also can trigger activation of the stimulator of interferon genes pathway to enhance the effects of ICIs independently of BRCA status. ${ }^{37}$

In the phase II MEDIOLA study, durvalumab (anti-PDL1) with olaparib (PARPi) combination was well tolerated in 32 patients with BRCA-mutated platinum-sensitive e relapsed ovarian cancer. The most common grade $\geq 3$ adverse events were anemia (9\%) increased lipase (9\%), hyperamylasemia (6\%) and neutropenia (3\%). The interim analysis showed a Disease Control Rate (DCR) based on RECIST at 12 weeks of $81 \%$ in case of co-administration of olaparib and durvalumab. The Complete Response (CR) and Partial Response (PR) rates were, respectively, 19\% and $44 \%$, resulting in an ORR of $63 \%$ while enhanced responses were seen in patients with only $1-2$ prior chemotherapies (ORR 69\%). ${ }^{38}$

Furthermore, in a phase I trial, the same combination resulted in an ORR of $17 \%$ and a DCR of $83 \%$ at 6 months according to RECIST in 12 patients with BRCA wild-type recurrent tumors and up to $>5$ lines of previous therapy, without dose-limiting toxicity (DLT). The authors concluded that immunotherapy plus PARPis can be considered a tolerable and clinically active treatment. ${ }^{32}$

The results of the phase I/II TOPACIO-KEYNOTE 162 trial have shown that the PARPi niraparib in combination with pembrolizumab (anti-PD-1) is tolerable. A promising antitumor activity was showed in particular for patients with platinum-resistant and platinumrefractory recurrent ovarian carcinoma, especially BRCAwild Type or non-Homologous Recombination Deficiency (HRD) disease, patients with limited treatment options (1-5 prior lines of therapy), regardless of platinum status, biomarker status, or prior treatment with bevacizumab. In the integrated efficacy phases 1 and 2 ovarian carcinoma population (60 of 62 evaluable patients), ORR was $18 \%$ (90\% CI, 11-29\%), with a disease control rate of $65 \%$ (90\% CI, 54-75\%), including 3 (5\%) with confirmed CR, 8 (13\%) with confirmed PR, 28 (47\%) with stable disease (SD), and 20 (33\%) with progressive disease (PD) based on RECIST. The most common adverse events of any grade $(\mathrm{n}=53)$ in Phase 2 were fatigue $(53 \%)$, nausea (42\%), anemia (36\%), and constipation (36\%). Adverse events of at least grade 3 were anemia (21\%) and thrombocytopenia $(9 \%)$. No treatment-related patient deaths or cases of myelodysplastic syndrome or acute myeloid 
leukemia occurred. ${ }^{39}$ Currently the study is active but no longer in the enrollment phase. ${ }^{40}$

Finally, a phase I study has been testing the combination of cediranib (VEGF receptor inhibitor), durvalumab (anti-PD-L1) and olaparib (PARPi) with an escalation in order to determine the recommended phase 2 dose (RP2D) while RRs, pharmacokinetic and correlative analyses were secondary endpoints. Nine patients ( 7 affected by recurrent platinum-resistant or sensitive OC or primary peritoneal cancer, median 3 prior therapies [2-6]) were treated. Adverse events (Grade 3/4) were not common and included hypertension (1/9), anemia (1/9) and lymphopenia (3/9) and none of these was a dose-limiting toxicity. The identified RP2D was cediranib $20 \mathrm{mg}$ (5 days on/2 days off) with full doses of durvalumab (1500 mg IV every 4 weeks) and olaparib (300 mg BID). The ORR was 44\% (4/9) with all PR (RECIST criteria), lasting a median of 8.5months [7-26 months]. Three patients had SD lasting $\geq 6$ months, yielding a $67 \%$ clinical benefit rate. No significant effects of the combination on pharmacokinetic were identified. ${ }^{41}$ A phase 2 expansion study is now enrolling for recurrent ovarian cancer patients. ${ }^{42}$

\section{Immunotherapy in Combination with Chemotherapy}

The standard chemotherapeutic approach for newly diagnosed ovarian cancer contemplates the combination of carboplatin and paclitaxel. ${ }^{43}$ This doublet has been associated successfully with PD-1 blockade in non-small-cell lung cancer (NSCLC), where the immunochemotherapy combination outperformed chemotherapy. ${ }^{44}$ Several authors studied carboplatin-paclitaxel and immunotherapy in newly diagnosed ovarian cancer with contradictory data. A randomized, open-label, multicenter, phase 3 study evaluated the efficacy (per RECIST v1.1) and safety of avelumab (anti-PD-L1) in combination and/or following chemotherapy in patients with previously untreated epithelial ovarian cancer (JAVELIN OVARIAN 100). The study was prematurely closed based on the results of a planned interim analysis that showed the futility of efficacy. The last results of December 2019 showed a PFS of 16.8 months in the group of chemotherapy followed by avelumab vs 18.1 months in arm treated with chemotherapy plus avelumab followed by avelumab vs NA (Not Available) (due to a limited number of events) in the group of chemotherapy alone plus observation. HR estimated were 1.43 (chemotherapy followed by avelumab vs chemotherapy alone plus observation) and 1.14 (chemotherapy + avelumab followed by avelumab vs chemotherapy alone plus observation) with $\mathrm{P}$ value $>0.05$ (NCT02718417). ${ }^{45}$

ICIs are not the unique immunotherapeutic tools that were tested in combination with upfront chemotherapy. A recent Phase II, randomized, the study evaluated carboplatin-paclitaxel chemotherapy in association with oregovomab, a high affinity murine monoclonal antibody specific for $\mathrm{Ca} 125$, in 97 cases of optimally resected, Stage III/IV disease. At a median follow up of 42 months, the study revealed an increase in PFS and OS of statistically and clinically significant magnitude: the median PFS was 41.8 months (95\% C.I.: 21.8 - NE Not Estimable) for the combination and 12.2 months (10.4-18.6) for the chemotherapy alone $(\mathrm{P}=0.0027$, HR 0.46 , CI $0.28-0.7)$. For OS, the median has not yet been reached (NE) in the oregovomab group (45.2-NE) while, in the other arm, it was of 43.2 months (31.8-NE) $(\mathrm{P}=0.043$, HR 0.35, CI 0.16-0.74). In addition, the administration of antibodies did not change the toxicity profile of standard chemotherapy. ${ }^{46}$

Simultaneously, efforts have been made in the evaluation of predictive factors for the response to treatment with oregovomab itself. Thus, Battaglia et al have recently assessed that efficacy of oregovomab is associated with a less suppressive immune environment at baseline: a low number of circulating myeloid-derived suppressor cells, subset type 4 (MDSC4), and a low neutrophil-andmonocyte to lymphocyte ratio (NMLR) were found in patients with a better response to treatment with oregovomab in addition to chemotherapy. Both parameters resulted significantly predictive for relapse-free survival (MDSC 4: $\mathrm{P}=0.012$, NMLR $\mathrm{P}=0.0014)$, while NMLR was related also with $\mathrm{OS}(\mathrm{P}=0.048){ }^{47}$

In case of recurrent ovarian cancer, several chemotherapeutic approaches are available, according to platinum sensitivity. In particular platinum-resistant or refractory disease represents the principal challenge for the oncologist. Among drugs available for these patients, guidelines cite weekly paclitaxel, pegylated liposomal doxorubicin (PLD), docetaxel, cyclophosphamide, gemcitabine and topotecan. ${ }^{43}$ Concerning the combination with immunotherapy, data showed that PLD could synergize with it: ${ }^{48}$ PLD would enhance tumor immunosurveillance and inhibit tumor immunosuppression but it would also synergize with immunotherapy improving indeed the sensitivity of tumor cells to 
the cytotoxic activity of natural killer (NK) cells, $\gamma \delta \mathrm{T}$ or CD ${ }^{+}$T lymphocytes. ${ }^{49}$

Several authors have addressed the issue of the administration of immunotherapy and chemotherapy in platinum-resistant relapses, with various results.

First of all, about the schedule with PLD in a phase II trial, the combination of durvalumab (anti-PD-L1) in 53 patients with recurrence after first or second-line platinumbased chemotherapy, resulted in a best ORR of $15 \%$, with a CR of 5\%, and PFS rate at 6 months of $47.7 \%$ with RECIST v1.1, leaning towards the hypothesis of a promising efficacy (NCT02431559). ${ }^{48,50}$

The preliminary results of another phase II study of PLD with pembrolizumab (anti-PD-1) in 26 women with platinum-resistant ovarian cancer (who received $\leq 2$ cytotoxic regimes for recurrent or persistent disease) were on the same line: the ORR and DCR were 19 and $42 \%$ respectively, with $5 \mathrm{PR}$ and $6 \mathrm{SD}$, defined as RECIST v1.1. The combination also resulted well tolerated with grade 3-4 adverse events of anemia (12\%), rash (12\%), and increased liver enzymes $(12 \%){ }^{51}$

On the opposite, in a phase II randomized study of 297 patients with recurrent EOC (Up to 2 prior cytotoxic regimens), the addition of a TLR8 agonist (motolimod) to PLD did not improve OS (log-rank one-sided P 0.923, HR 1.22) and PFS (log-rank one-sided P 0.943, HR 1.21) assessed by the Immune-Related RECIST (irRECIST) criteria. ${ }^{52}$ Similarly, the phase III JAVELIN Ovarian 200 study compared PLD with the combination of PLD and the anti-PD-L1 antibody avelumab in platinum-resistant /refractory ovarian cancer received up to 3 lines of systemic anticancer therapy for platinum-sensitive disease and no prior systematic therapy for platinum-resistant disease. Authors concluded that the combination of PLD+ avelumab did not show a statistically significant improvement when compared with PLD alone in PFS (RECIST) (HR 0.78; CI 0.587-1.244; $\mathrm{P}=0.0301$ ) or OS (HR 0.89; CI $0.744-1.241 ; \mathrm{P}=0.2082$ ) (NCT02580058). ${ }^{53}$ The study closed the accrual, and definitive results are awaited.

Finally, some authors proved that also low-dose intravenous or oral metronomic chemotherapy can have important immunomodulatory effects. Between these, low-dose cyclophosphamide has been shown to attenuate Tregs and improve vaccination as well as adoptive cell therapy (ACT) efficacy, ${ }^{54}$ whereas, in combination with ICIs, it stimulates the generation of $\mathrm{CD} 8^{+}$TILs. ${ }^{55}$ Thus, cyclophosphamide was evaluated in recurrent ovarian cancer itself. In particular, an open-label phase II study has been testing the combination of cyclophosphamide with bevacizumab (anti-VEGF) and pembrolizumab (anti-PD-1) in 40 patients with recurrent $\mathrm{OC}$, platinum-resistant or platinumsensitive refusing platinum-based therapy regardless of the number of previous chemotherapies. This has been evaluating the clinical RRs using both RECIST1.1 and irRECIST. ${ }^{56}$ To a preliminary analysis, the combinations were well tolerated and it was found that the tumor responses were higher and longer than those obtained with monotherapy: the ORR was $37.5 \%$ (all PR), and the overall PFS rate was $70 \%$ at 6 months $(59 \%$ in the platinum-resistant population, $100 \%$ in platinum-sensitive ones; $\mathrm{P}=0.024){ }^{57}$

To sum up, some authors proofed that the combination of chemo-immunotherapy could improve the efficacy of the treatment, even in $\mathrm{OC}$ with the worst prognosis, but data are not universally consistent. Thus, further studies are needed to establish it with certainty.

\section{Other Combinations}

Pembrolizumab (anti-PD-1) combined with mirvetuximab soravtansine (a folate receptor-a [FRa] antibody-drug conjugate that comprises an FRa-binding antibody linked to the tubulin-disrupting maytansinoid DM4) also has shown preliminary signs of efficacy in a heavily pre-treated population (no upper limit on the number of prior treatments) of 14 FRa-positive patients with platinum-resistant ovarian cancer in the phase Ib/II FORWARD II trial: In the subset of 8 patients with medium to high FRa expression levels by immunohistochemistry, confirmed PR were observed in 3 individuals according to RECISTv1.1 and CA125 evaluations; 2 were ongoing with at least 5 months on treatment; and the third had a duration of therapy of 7 months. ${ }^{58}$ These findings supported the ongoing enrollment of patients in an expansion cohort. ${ }^{59}$

\section{Combinations of Immune-Checkpoint Inhibitors}

Combining the activation of different mechanisms of the immune system can achieve more pronounced antitumor activity than blockade of a single pathway alone.

As good evidence of this, Co-targeting of CTLA-4 (ie ipilimumab) plus PD-1 (ie nivolumab) resulted in significantly stronger activity in non-gynecological tumors ${ }^{60}$ due to the synergism of amplification of T-cells in lymphoid organs and tumor tissue by anti-CTLA-4, and PD-1 inhibition that overcomes immune suppression in tumor tissues. 
An NRG Oncology phase II study has been testing these agents together in a population of 100 patients with recurrent ovarian cancer with up to 3 prior lines of therapy and a platinum-free interval of up to 12 months. It reported ORR by RECIST v1.1 of $33.3 \%$ and PFS 3.9 months for the combination vs ORR $12.2 \%$ and PFS 2.0 months with nivolumab alone. Adverse events occurred more frequently in the combination arm (Grade 3 or greater AEs in $67 \%$ ) versus the single-agent arm (Grade 3 or greater AEs in 55\%). ${ }^{61}$ The trial is no more recruiting but still ongoing, analysing new biomarkers for response. ${ }^{62}$

A phase $\mathrm{I} / \mathrm{ll}$ dose escalation and cohort expansion trial is testing the safety, tolerability and efficacy of varlilumab (an anti-CD27 antibody) and nivolumab (anti-PD-1) in advanced refractory solid tumors among which eight cases of ovarian cancer. Overall, upon an initial analysis, the combination was well tolerated, associated with strong biological signals, and had evidence of clinical activity (based on RECIST) in subsets of patients with tumor types resistant to PD-1 inhibitor monotherapy. ${ }^{63} \mathrm{Up}$ to now results from phase 2 cohort in ovarian cancer are awaited. $^{64}$

All in all, these data, although preliminary and coming from phase I-II studies, are promising.

\section{Other Strategies Adoptive Cell Therapy}

Adoptive Cell Therapy (ACT) is an immunotherapeutic technique that uses autologous or allogeneic antitumor lymphocytes to induce regression in cancers that are less responsive or refractory to ICIs.

The approach of autologous ACT is illustrated in Figure 1 and involved cell types are summarized in Table $1 .{ }^{65}$

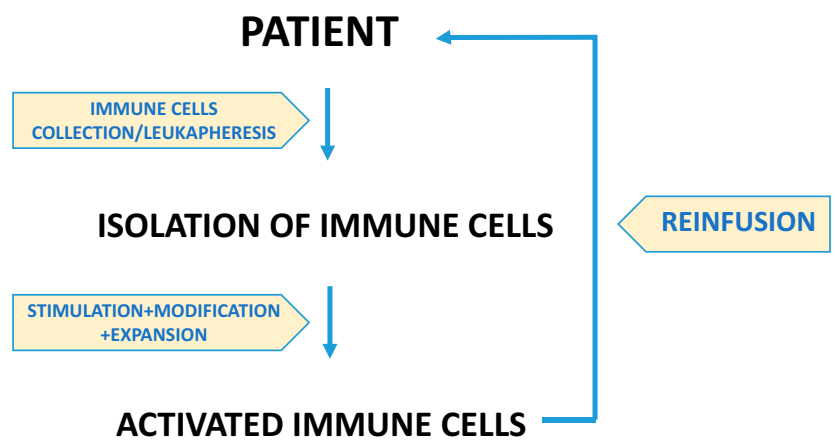

Figure I Autologous ACT is based on reinfusion of immune cells after stimulation, modification and expansion in vitro in order to amplify autologous response against tumors. These tumor-specific cytotoxic T cells, either isolated from the tumor or in the peripheral blood by leukapheresis, are then infused after lymphodepleting chemotherapy.
The potential role of Natural Killer (NK) cell adoptive immunotherapy for OC was recognized in 2007 when it was shown that resting NK cells can recognize and kill OC cells in vitro ${ }^{66}$ and subsequently, in 2016, a new mouse model was established for intraperitoneal administration of NK cell immunotherapy for OC allowing further research. ${ }^{67}$

A phase II study analyzed the role of another class involved in ACT, the Cytokine-Induced Killer (CIK) cells, in the approach to OC: Liu et al tested this therapy versus observation in 92 patients with completed remission after first-line treatment for advanced OC. The median PFS was 37.7 months in the treatment group and 22.2 months in the control group $(\mathrm{P}=0.004)$ with slight side effects, although with a no significant difference in OS (61.5 months vs 55.9 months, $\mathrm{P}=0.289){ }^{68}$

In 2018, a retrospective analysis by Zhou et al further sustained the effectiveness of maintenance CIK cell therapy as a therapeutic approach to prolonging the survival in 646 OC patients after first-line treatment. Specifically, patients who received CIK exhibited a significantly more favourable OS than control group patients (median OS, 63.6 vs 39.6 months, $\mathrm{P}=0.001$ ), while Kaplan-Meier curves showed a favourable PFS (median PFS, 41.6 vs 26.1 months), but with the not-significant $\mathrm{p}$-value $(\mathrm{P}=0.117) .{ }^{69}$

Referring to TILs, the first data date back to before the end of the $90 \mathrm{~s}$.

Some of the most relevant results obtained with autologous TILs are summarized in Table $3 .^{70-73}$

The therapeutic effect of TILs in OC is still under evaluation.

In 2018, results from a pilot study in platinum-resistant recurrent OC with lympho-depleting chemotherapy followed by the infusion of unselected TILs and IL2 were published. Clinical response was assessed using the RECIST 1.1 criteria but the Positron Emission Tomography (PET) Response Criteria in Solid Tumors (PERCIST 1.0) was studied in order to identify the difference between the two models. The therapy was feasible and with no unexpected toxicities related and early indications of activity were found: all patients had SD after 6 weeks and five of them had a reduction of target lesions. Two patients maintained this response for 5 months. Subsequently, five patients developed PD due to the occurrence of new lesions. In this way, the road toward the possibility of enhancing ACT combining it to ICIs was opened. $^{74}$ Subsequently, the same group managed to expand TILs from 34 tumors specimen of OC and found the recognition of autologous tumor cell in $>50 \%$ of the 
Table 3 Some Relevant Results of ACT Obtained Up to 2000 with Autologous TILs

\begin{tabular}{|c|c|}
\hline Author/Year & Results of Study \\
\hline Aoki et $\mathrm{al}^{70}$ & $\begin{array}{l}\text { - } 17 \text { patients } \\
\text { - Advanced or Recurrent OC } \\
\text { - Treatment: TILs } \pm \text { cisplatin } \\
\text { - Combination (I0 pts) } \rightarrow \text { CR } 70 \%, \text { PR } 20 \% \\
\text { - Monotherapy (7pts) } \rightarrow \text { CR } 14.3 \%, \text { PR } 57.1 \%\end{array}$ \\
\hline $\mid k a r a s h i$ et $\mathrm{al}^{71}$ & $\begin{array}{l}\text { - } 22 \text { patients } \\
\text { - Advanced EOC (FIGO II-III-IV) } \\
\text { - Treatment: TILs } \\
\text { - TILs group (I2 pts) } \rightarrow \text { Immunoactivation of cellu- } \\
\text { lar immunity, } 2 \text {-year survival rate: } 100 \% \\
\text { - No-TILs group ( } 10 \text { pts) } \rightarrow \text { No changes in immu- } \\
\text { nological markers, } 2 \text {-year survival rate: } 100 \%\end{array}$ \\
\hline $\begin{array}{l}\text { Freedman } \\
\text { et } \mathrm{al}^{72}\end{array}$ & $\begin{array}{l}\text { - Il patients } \\
\text { - Advanced EOC refractory to platinum-based } \\
\text { chemotherapy } \\
\text { - Treatment: IL2 ip } \pm \text { TILs } \\
\text { - IL2+TILs group }(8 \mathrm{pts}) \rightarrow \text { No measurable clinical } \\
\text { response. } 4 / 8 \text { secondary response } \\
\text { - IL2 group ( } 3 \text { pts }) \rightarrow \text { No measurable clinical } \\
\text { response }\end{array}$ \\
\hline Fujita et $\mathrm{al}^{73}$ & $\begin{array}{l}\text { - } 24 \text { patients } \\
\text { - EOC with CR after first-line chemotherapy } \\
\text { - Treatment: TILs } \\
\text { - TILs group (I } 3 \text { pts) } \rightarrow 3 \text {-year survival rate } 100 \% \text {, } \\
\text { - No-TILs group (I l pts) } \rightarrow 3 \text {-year survival rate } 67.5 \%\end{array}$ \\
\hline
\end{tabular}

Abbreviations: $C R$, complete response; EOC, epithelial ovarian cancer; IP, intraperitoneal; OC, ovarian cancer; PR, partial response; PTS, patients; TILs, gtumorinfiltrating lymphocytes.

patients. Furthermore, they isolated and subsequently expanded antigen-specific TILs. These findings support the hypothesis that women with $\mathrm{OC}$ can benefit from ACT with TILs and encourage further studies. ${ }^{75}$ Anyway, these studies revealed some of the potential limitations of TILs-ACT in recurrent EOC that have to be overcome: the exhausted phenotype of TILs ${ }^{74}$ and/or the low frequency of tumor-reactive TILs in the infusion product. ${ }^{75}$

With the aim of improving the therapeutic efficacy, genetically modified peripheral blood lymphocytes have been being studied with more interest (Figure 2). They can express a chimeric antigen receptor (CAR) or a tumorantigen specific T-cell receptor (TCR).

The main targets for CAR-T cells in OC include MUC16 (Mucin 16)/Ca 125, mesothelin and folate receptor- $\alpha^{76-78}$ while for TCR, they are MAGE-A4 (Melanoma-associated antigen 4), WT1 (Wilms' tumor protein 1), and NY-ESO-1 (New York oesophageal-1). Among them the last one is the most widely studied and proved to be overexpressed not only in OC but also in all gynecological cancers. ${ }^{79-82}$

The main evidences from experimental animal models and clinical data on CAR-T and TCR-T role in OC are illustrated in Table $4 .^{83-87}$

Although their administration has shown a potential in the treatment of $\mathrm{OC}$, it still has to face many challenges. Currently, several phase I/II clinical trials are ongoing.

\section{Therapeutic Vaccine}

Cancer vaccines are immunotherapeutic agent, usually a tumoral antigen, that stimulate an immune response ( $\mathrm{T}$ cells) directed against specific malignant cells. ${ }^{88}$

A number of tumor-associated antigens for vaccine have been identified in EOC. The ideal ones should not be present in normal tissue, while expressed at high concentration in tumor ones, having a role in its progression. Moreover, it should be able to be recognized by immune cells and activate the immune system response event at low doses, characteristics known as "strong immunogenicity". None of the currently known antigens, however, completely meet these criteria.

Anyway, among antigens possibly involved for immunotherapy in OC, the cancer-testis (CT) family is quite wide and includes the NY-ESO-1. Several trials evaluating NY-ESO -1-based vaccine have been conducted in the treatment of EOC, in different schedules. Data from these trials showed a favourable efficacy, with an improved OS of at least 2 years in case of vaccination in both newly diagnosed or recurrent OC. ${ }^{48,89-93}$ Currently other several single antigens, eg MUC-1 (Mucin 1), mesothelin, HER2 (human epidermal growth factor receptor), MUC-16/Ca125, p53, Testis Expressed 19 (TEX 19) ${ }^{94}$ can be seen as a possible target of vaccines for OC.

Moreover, since cancer vaccination has the purpose to educate the immune system to generate specific effector T-cells able to detect and kill tumor cells, dendritic cell-based vaccines are a particularly attractive option for immunotherapy, due to the capability of these cells to process and present cancer antigens, and to initiate and regulate both innate and adaptive immunity. ${ }^{95,96}$ A phase II trial tested a MUC-1 targeted Dendritic Cell treatment (CVac) as a maintenance therapy versus observation to 56 patients in complete response after a first or second-line chemotherapy. Although a response was induced in all CVac-treated based on RECIST v1.1, the effect on PFS was not significant in 


\section{PATIENT}

\begin{tabular}{|c|}
\hline IMMUNE CELLS \\
COLLECTION/LEUKAPHERESIS \\
\hline
\end{tabular}

ISOLATION OF IMMUNE CELLS

STIMULATION+MODIFICATION+
EXPANSION:
Activation and Amplification

\section{ACTIVATED IMMUNE CELLS}

\section{REINFUSION}

\section{AMPLIFICATION + QUALITY CONTROL}

GENETIC ENGINEERING:

Receptor Gene Transfer

\section{CAR-T/TCR-T}

Figure 2 Immune cells (T cells) are isolated from patients. They are activated and amplified in vitro and then modified by genetic engineering (receptor gene transfer through viral vector transfection). After amplification and quality control, CAR-T cells and TCR-T cells are reinfused to the patient.

the treated arm compared with the control one (13 immunogenicity. They arise from somatic DNA alterations months vs 9 months, $\mathrm{P}=0.36$, HR 0.73$).{ }^{97}$

Alternatively, the so-called neoantigens (NeoAgs) could ensure high specificity, reducing the potential for immunological tolerance, and inducing robust as a result of genetic instability that generate peptides entirely absent from the normal human genome. For these reasons, they are cancer-specific and strongly immunogenic. $^{98}$

Table 4 Evidence from Experimental Animal Models and Clinical Data on TCR-T and CAR-T Role in OC

\begin{tabular}{|c|c|c|c|}
\hline Author/Year & Type of Act & Mice/Women & Conclusion \\
\hline $\begin{array}{l}\text { Chekmasova et al } \\
2010\end{array}$ & $\begin{array}{l}\text { CAR-T MUC } 16 \\
\text { IP }\end{array}$ & OC Mice & $\begin{array}{l}\text { - } 100 \% \text { of mice: eradication of most evident disease } \\
\text { - } 75 \% \text { of mice: relapse at a later time } \\
\text { - } 25 \% \text { of mice: NED after } 120 \text { days. }\end{array}$ \\
\hline Carpenito et $\mathrm{al}^{84}$ & $\begin{array}{l}\text { CAR-T mesothelin } \\
\text { IV } \\
\text { IP } \\
\text { IT }\end{array}$ & OC Mice & $\begin{array}{l}\text { - Therapy reduced the tumor burden ((even complete eradication) } \\
\text { - IT injection is marginally faster than IV but significantly better than IP. }\end{array}$ \\
\hline Tanyi et al ${ }^{85}$ & $\begin{array}{l}\text { CAR-T mesothelin } \\
\text { IV }\end{array}$ & $\begin{array}{l}6 \text { Women } \\
\text { Recurrent OC }\end{array}$ & $\begin{array}{l}\text { - Clearing of malignant cells in the pleural fluid (I patient) } \\
\text { - } 100 \% \text { SD at I monthh after treatment (RECIST and mRECIST criteria) }\end{array}$ \\
\hline Song et $\mathrm{al}^{86}$ & $\begin{array}{l}\text { CAR-T FR } \alpha \\
\text { IV } \\
\text { IP } \\
\text { IT }\end{array}$ & OC Mice & $\begin{array}{l}\text { - CAR-T cells eradicate large pre-established tumors in vivo } \\
\text { - IV and IP: activity delayed in regression by } \sim 7 \text { days relative to IT }\end{array}$ \\
\hline Anderson et $\mathrm{al}^{87}$ & $\begin{array}{l}\text { TCR-T } 1045 \\
\text { IV }\end{array}$ & OC Mice & $\begin{array}{l}\text { - Vaccine+TCR } 1045 \text { T cells Group: TTP II2 days } \\
\text { - } \text { TCR }_{1045} \text { T cells Group: TTP } 9 \text { I days } \\
\text { - No-Treatment Group: TTP } 77 \text { days }\end{array}$ \\
\hline
\end{tabular}

Abbreviations: ACT, adoptive cell therapy; CAR, chimeric antigen receptor; FR $\alpha$, folate receptor $\alpha$; IP, intraperitoneal; IT, intratumoral; IV, intravenous; mRECIST, modified response evaluation criteria in solid tumors; NED, no evidence of disease; OC, ovarian cancer; RECIST, response evaluation criteria in solid tumors; SD, stable disease; TCR, T cell receptor; TTP, time to progression. 


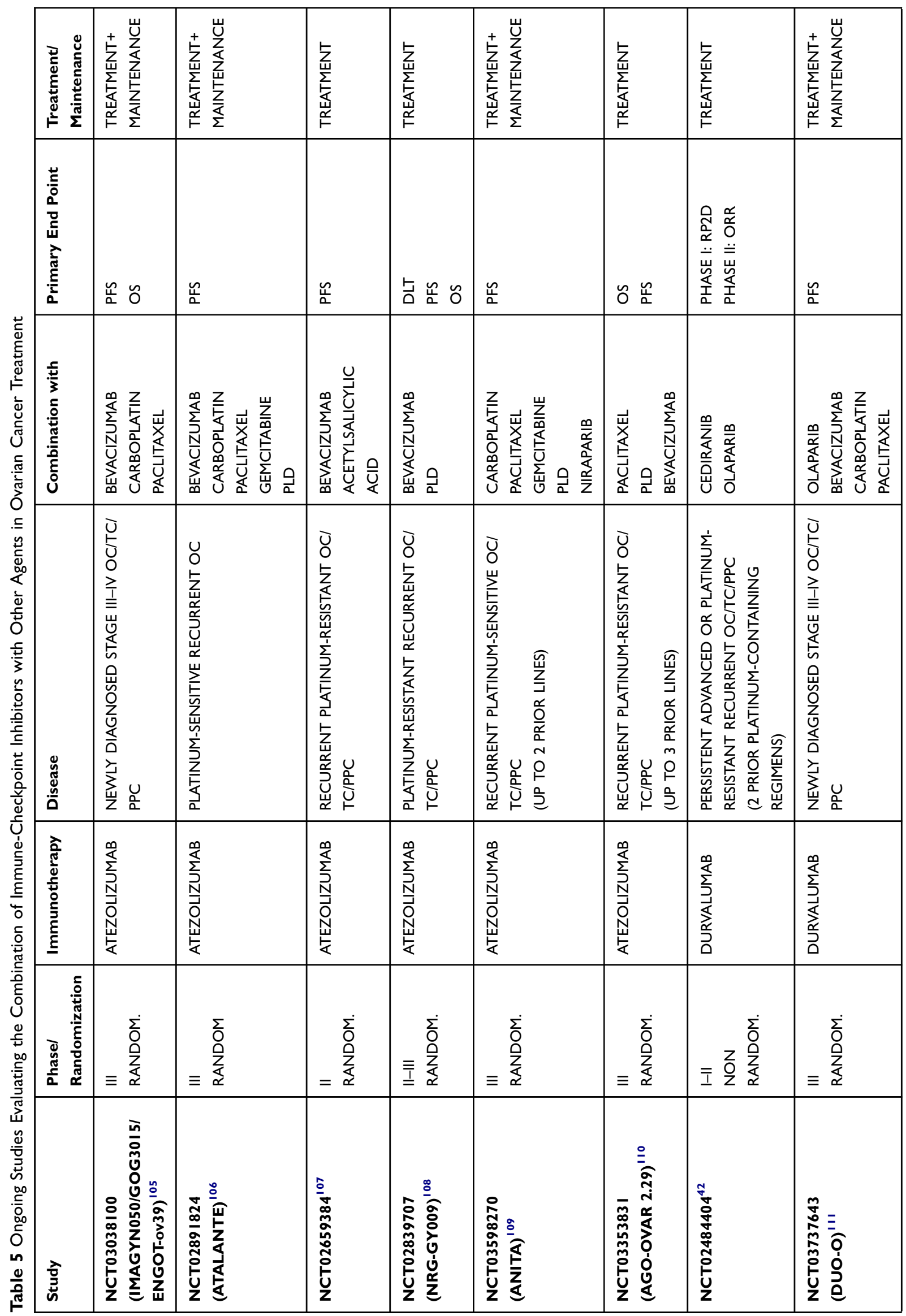




\begin{tabular}{|c|c|c|c|c|c|c|c|c|c|c|}
\hline 点 & 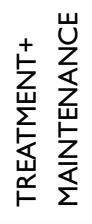 & 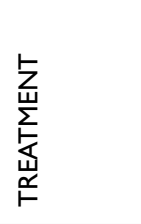 & 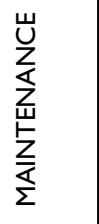 & 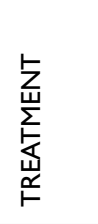 & 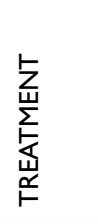 & 崖 & 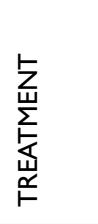 & 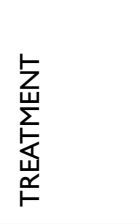 & 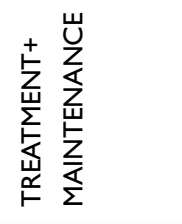 & 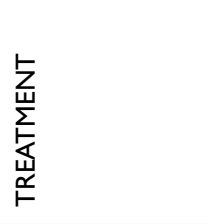 \\
\hline 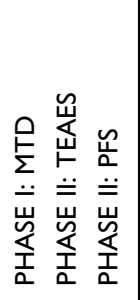 & 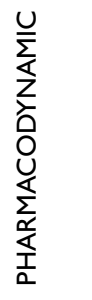 & $\begin{array}{l}\stackrel{\mathscr{\alpha}}{o ̛ ~} \\
0\end{array}$ & 씀 & 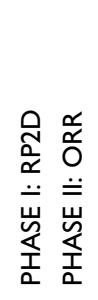 & 岁 & 씀 & ŏ & 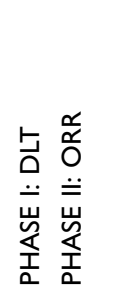 & 뜸 气ั & 峞ち \\
\hline 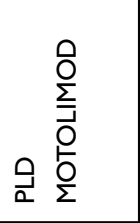 & 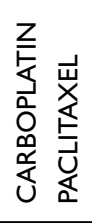 & 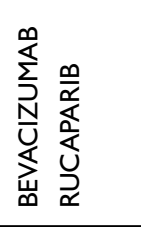 & $\begin{array}{l}\frac{0}{\alpha} \\
\frac{\alpha}{\alpha} \\
\frac{\alpha}{\alpha} \\
\grave{\alpha}\end{array}$ & $\begin{array}{l}\frac{9}{\alpha+\frac{\alpha}{\alpha}} \\
\frac{1}{\Delta} \\
0\end{array}$ & $\begin{array}{l}\frac{m}{\alpha} \\
\frac{\alpha}{\alpha} \\
\frac{\Delta}{\Delta} \\
0\end{array}$ & 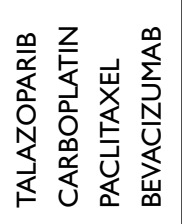 & $\stackrel{a}{a}$ & $\begin{array}{l}\frac{\infty}{\alpha} \\
\frac{\alpha}{\alpha} \\
\frac{\alpha}{\partial z}\end{array}$ & 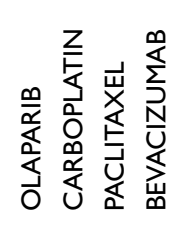 & 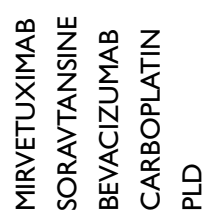 \\
\hline 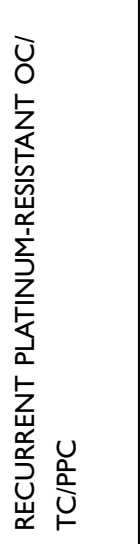 & 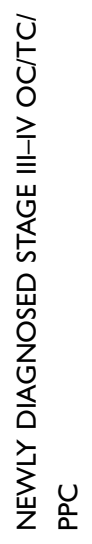 & 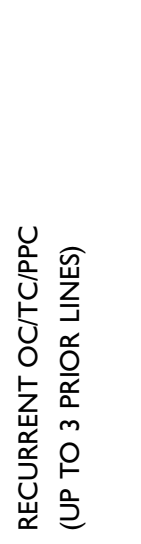 & 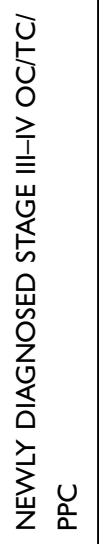 & 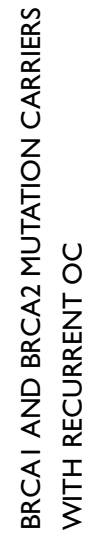 & 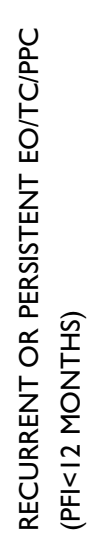 & 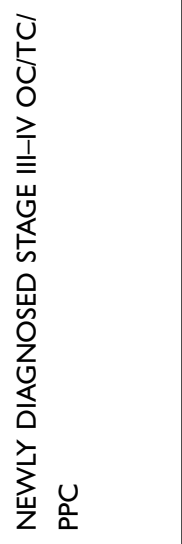 & 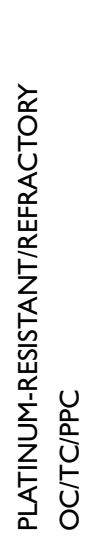 & 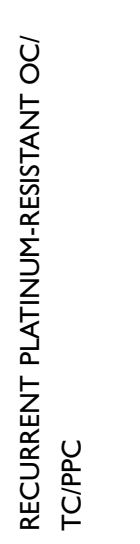 & 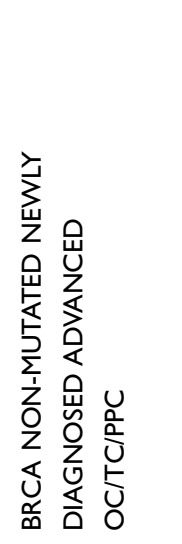 & 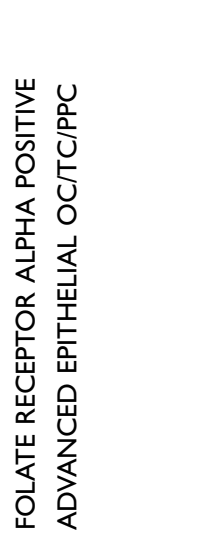 \\
\hline 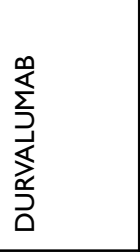 & 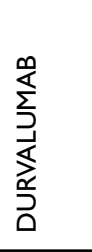 & 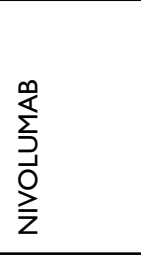 & $\begin{array}{l}\frac{\infty}{s} \\
\sum_{\supset}^{0} \\
\stackrel{\partial}{z}\end{array}$ & 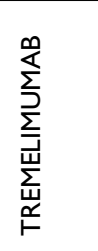 & 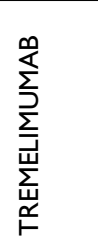 & 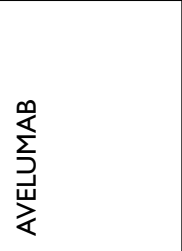 & 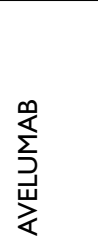 & 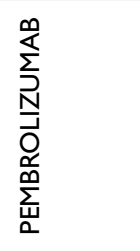 & 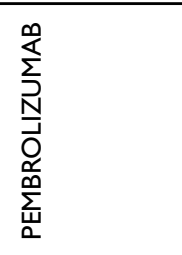 & 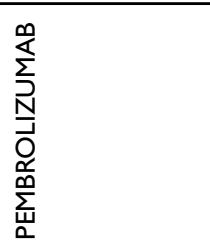 \\
\hline 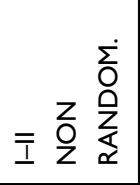 & 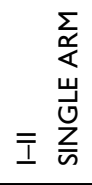 & 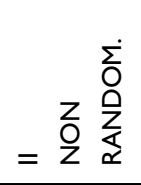 & 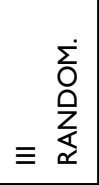 & 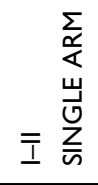 & 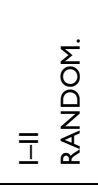 & 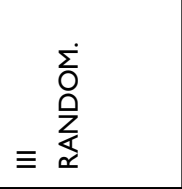 & 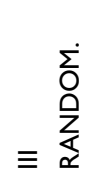 & 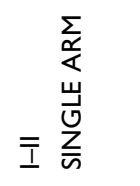 & 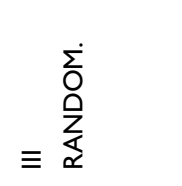 & $=\frac{\substack{\dot{\Sigma} \\
\mathrm{Z}}}{\mathrm{I}} \mathrm{Z} \underset{\nwarrow}{\mathrm{Z}}$ \\
\hline 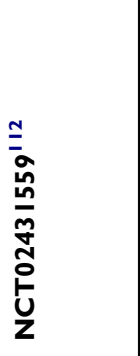 & 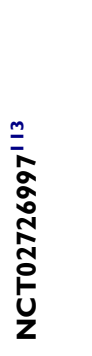 & 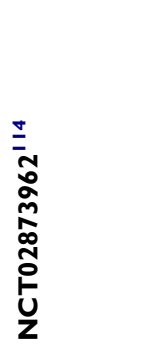 & 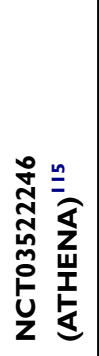 & 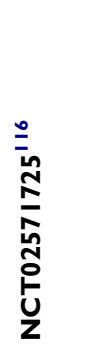 & 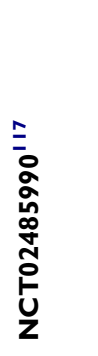 & 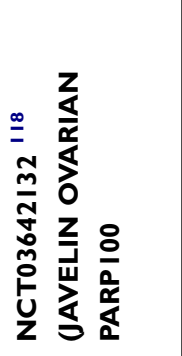 & 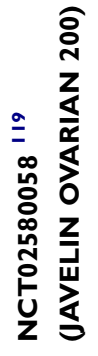 & 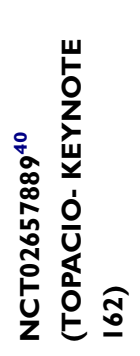 & 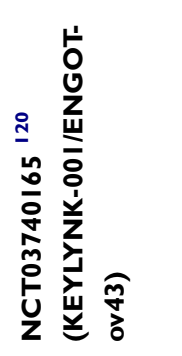 & 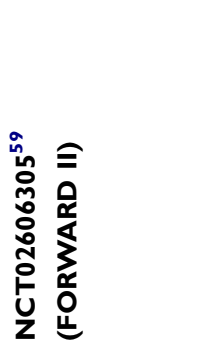 \\
\hline
\end{tabular}




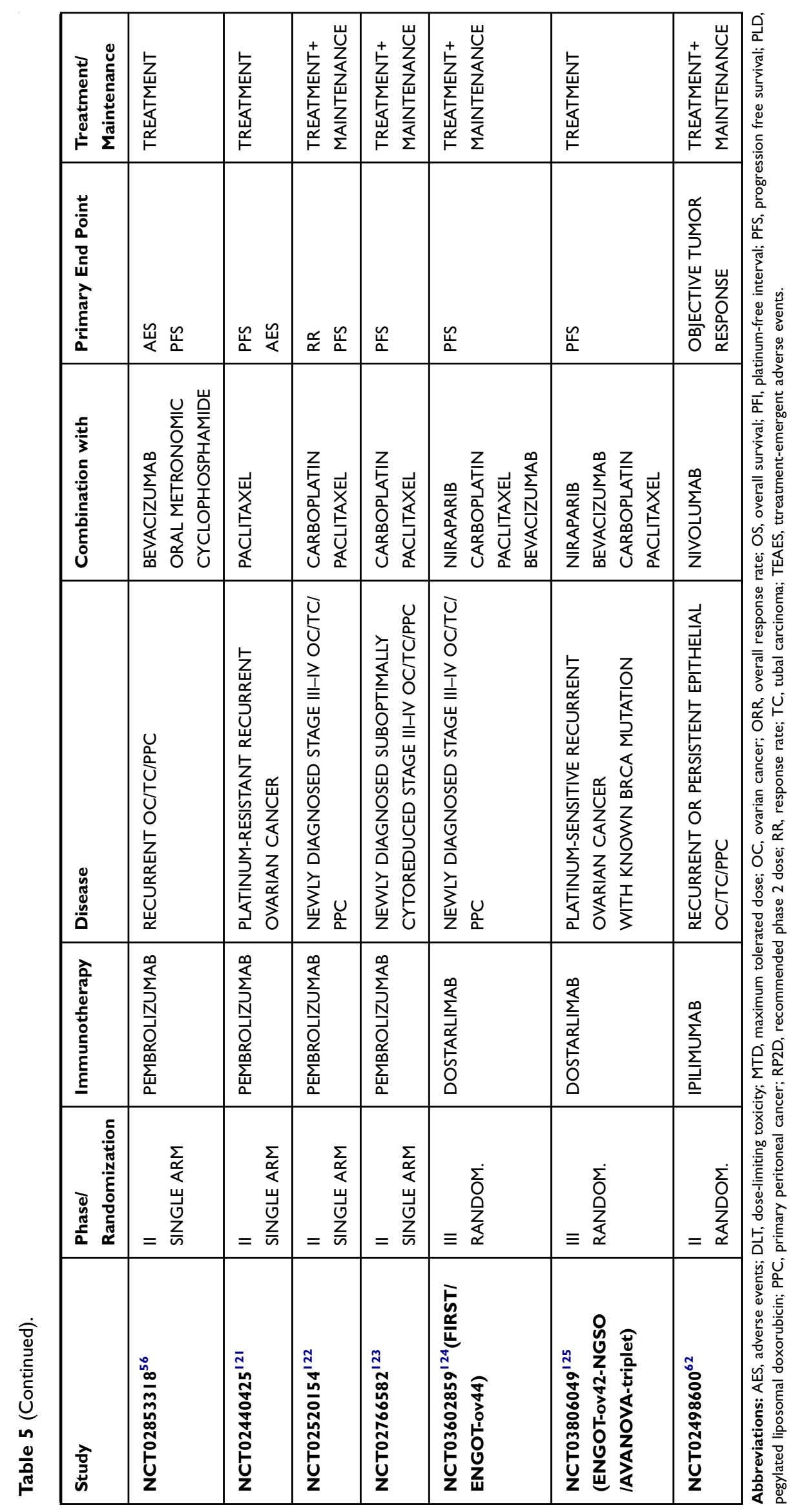




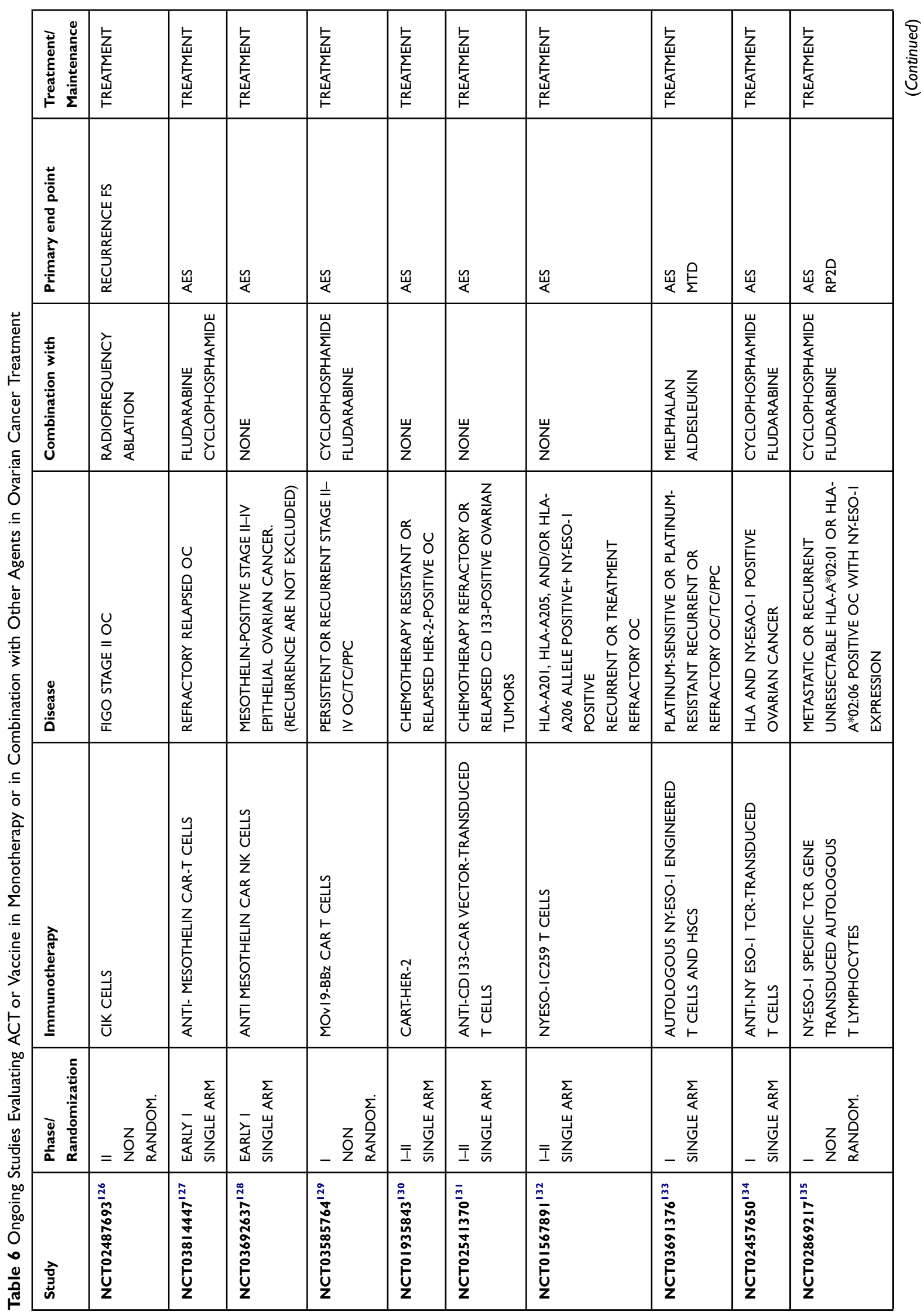




\begin{tabular}{|c|c|c|c|c|c|c|c|c|c|}
\hline 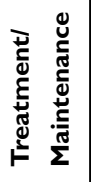 & 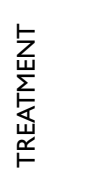 & 点 & 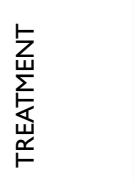 & 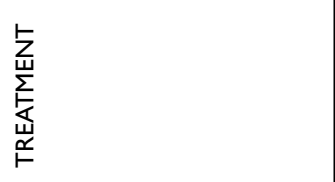 & 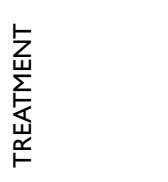 & 点 & 点 & 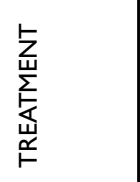 & 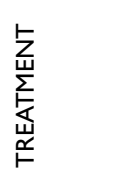 \\
\hline 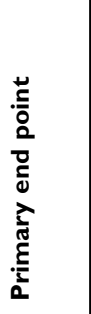 & 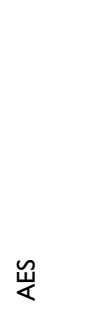 & 亗 & $\frac{\rho}{\Sigma}$ & 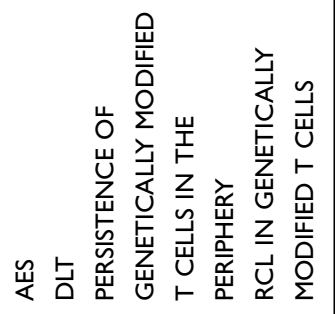 & $\frac{\alpha}{\alpha}$ & $\stackrel{5}{\Delta}$ & $\frac{\mathscr{\alpha}}{\widetilde{\alpha}}$ & 岀 总 & 岁 \\
\hline 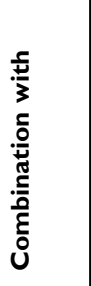 & $\begin{array}{l}\text { 岁 } \\
\text { O }\end{array}$ & 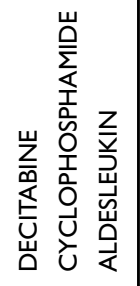 & 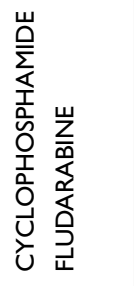 & $\begin{array}{l}\text { Uు } \\
\text { O }\end{array}$ & 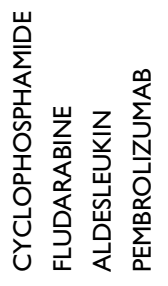 & 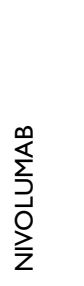 & 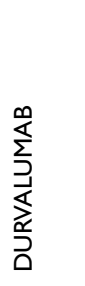 & 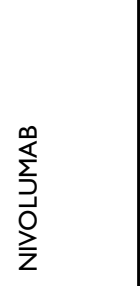 & 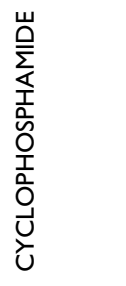 \\
\hline 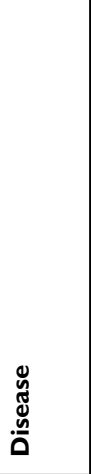 & 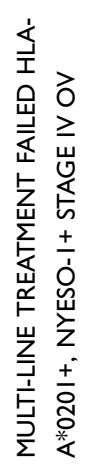 & 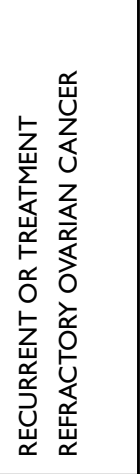 & 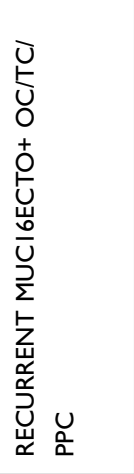 & 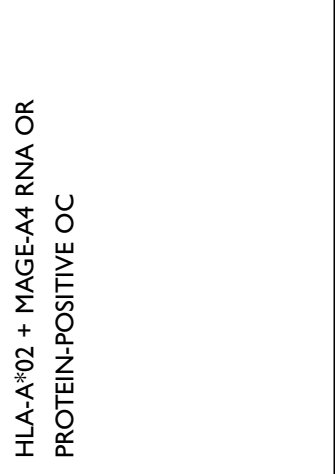 & 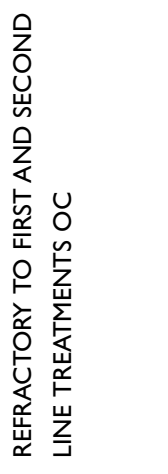 & 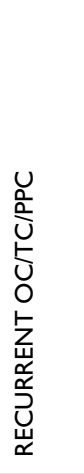 & 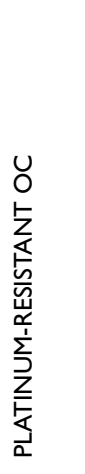 & 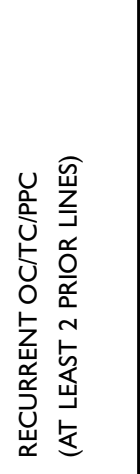 & 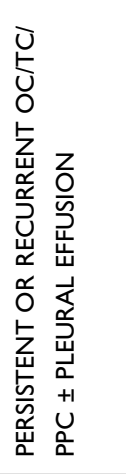 \\
\hline 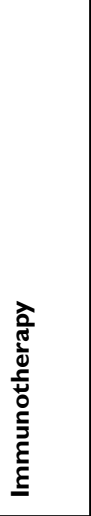 & 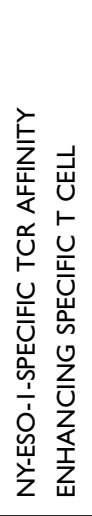 & 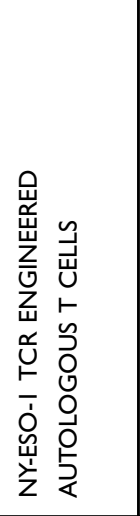 & 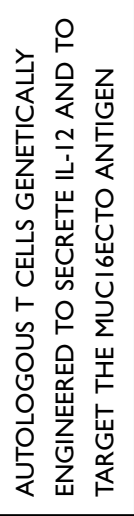 & 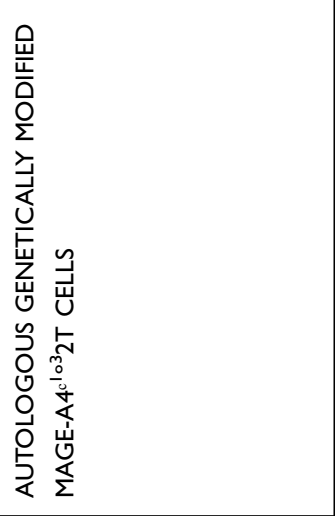 & 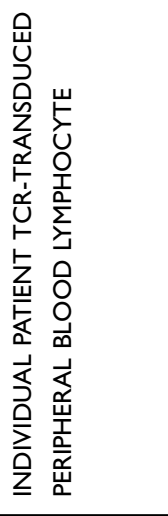 & 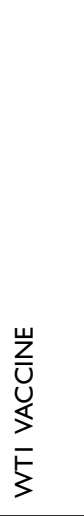 & 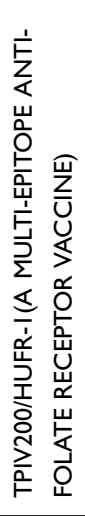 & 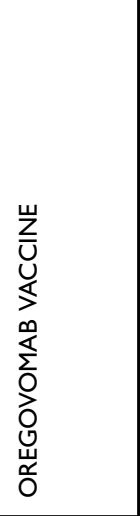 & 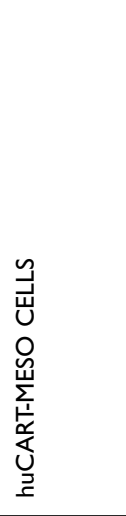 \\
\hline 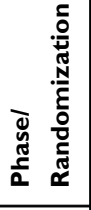 & 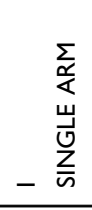 & 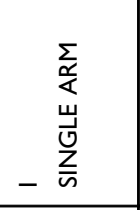 & 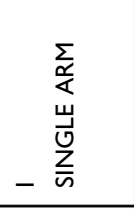 & 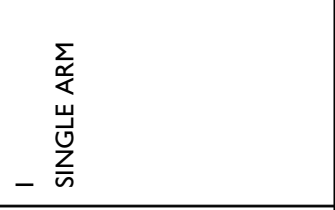 & 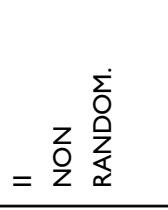 & 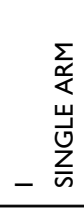 & 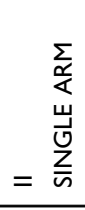 & 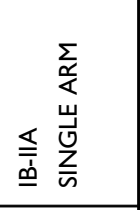 & $\begin{array}{r}\sum \\
z \\
-\mathrm{O} \\
-\mathrm{z} \\
\end{array}$ \\
\hline 窟 & 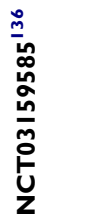 & $\begin{array}{l}\frac{\bar{m}}{\bar{m}} \\
\frac{n}{0} \\
\overline{\tilde{n}} \\
\frac{\mathfrak{L}}{z}\end{array}$ & 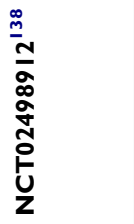 & 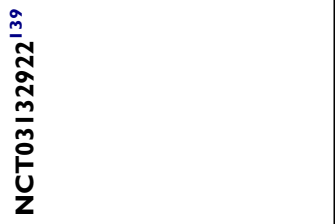 & 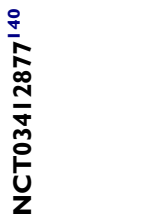 & 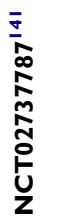 & 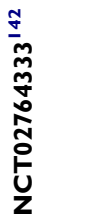 & 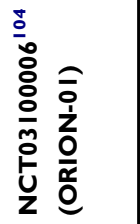 & 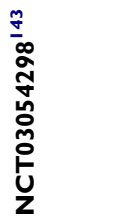 \\
\hline
\end{tabular}




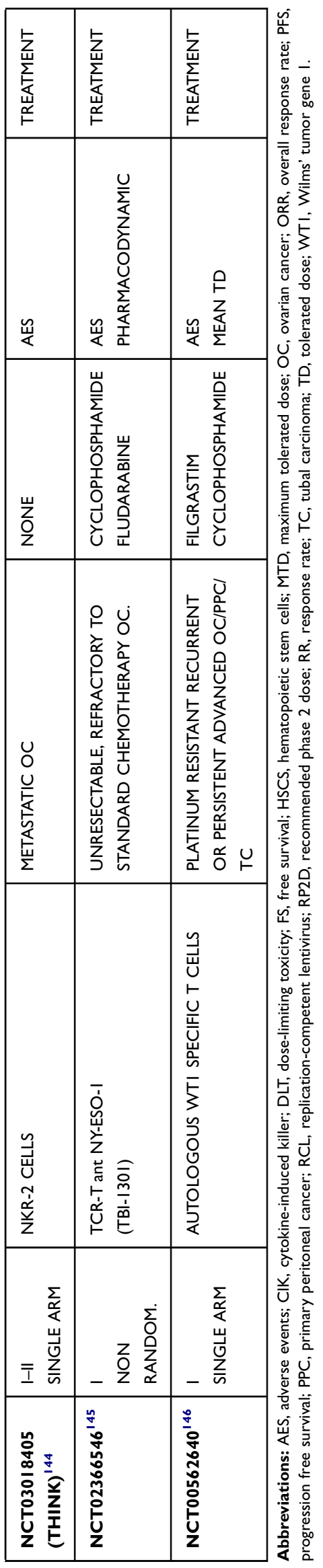

Nevertheless, they are hard to identify due to their patient-specificity itself and are mainly rare events in a patient cohort. ${ }^{99}$

Anyway vaccination efficacy could be compromised by tumor through the selection of antigen-loss variants (immunoediting) and the up-regulation of immunecheckpoint ligands as an escape mechanism. ${ }^{100,101}$ Thus, multiple vaccine interventions that activate de novo immune response may not appear to induce tumor regression. ${ }^{101}$ In order to overcome this limit, preclinical studies have observed an effective synergy between tumor vaccines and the ICIs. Hence, combining these two approaches may improve the efficacy in patients that would not have responded to either therapy alone. ${ }^{102}$

As a starting point, a phase Ib trial determined the Recommended Dose for Expansion (RDE)/RP2D of the association of oregovomab vaccine and nivolumab to treat recurrent EOC. ${ }^{103}$ Further evaluation of this novel combination is ongoing in a dose expansion cohort. ${ }^{104}$

\section{Ongoing Studies}

Immunotherapy in combination with other agents, ACT and vaccine are currently being examined in several ongoing clinical trials for newly diagnosed or recurrent ovarian cancer. Their characteristics are summarized in Tables 5 and 6 .

\section{Conclusion}

Immunotherapy has transformed cancer treatment in certain solid malignant tumor, such as melanoma and renal cell carcinoma. EOC is considered an "inflamed tumor" that could benefit from these agents. However, data are not universally consistent since the majority of studied patients with OC did not benefit from immunotherapy and there are some issues to be addressed.

First of all, it should be considered that many of clinical trials about immunotherapy and OC involved a population of women with a poor prognosis (advanced FIGO stage, platinum-resistant or -refractory disease, numerous previous chemotherapy lines) in which tumor growth and progression are associated with a more pronounced immune suppression. Hence, it remains still unclear at what phase of disease it would be more appropriate to administer immunotherapy.

Moreover, not all the patients with $\mathrm{OC}$ do respond to immunotherapy similarly, thus efforts have recently been being paid worldwide to identify some predictive biomarkers of efficacy, including the expression of antigens. 
Regarding that, it is important to clarify that EOC is a tumor with high genetic instability. So the evaluation of molecular markers on the tissue obtained during a previous surgery could exclude patients from accessing target immunotherapies subsequently. Thus, a promising tool could be represented by the liquid biopsy: with a noninvasive approach, it can identify blood-circulating cancer cells and their antigens that represent potential biomarkers.

Another difficulty in administrating immunotherapy concerns the evaluation of the response of solid tumors to this class of agents. As a matter of fact, this treatment may lead to atypical patterns characterized by delayed tumor size reduction, mixed response, or the so-called pseudoprogression: an initial tumor burden increase (growing of lesion size and/or appearance of new lesions) with subsequent decrease $^{147}$ probably due to immune cells infiltration. This led to the development of new special criteria to evaluate the efficacy of immunotherapy, the Immune-RECIST. Unfortunately, not all clinicians have already gotten used to understanding and using these criteria, ${ }^{148}$ despite the drafting of guidelines in $2017 .{ }^{149}$ At last, the possibility of immune-related adverse events (pneumonitis, colitis, thyroiditis and pancreatitis) must be considered.

To sum up, this review does not want to establish whether immunotherapy is active in the treatment of OC but the available results of evidence about combinations, ACT or vaccination are encouraging.

Currently, the administration of immunotherapy in OC is possible only in the context of clinical trials but we expect that ongoing phase II-III trials will untie all these knots, leading to important progress in OC treatment.

\section{Disclosure}

The authors report no conflicts of interest in this work.

\section{References}

1. Siegel RL, Miller KD, Jemal A. Cancer statistics, 2019. CA Cancer J Clin. 2019;69(1):7-34. doi:10.3322/caac.21551

2. Perren TJ, Swart AM, Pfisterer J, et al. A phase 3 trial of bevacizumab in ovarian cancer. $N$ Engl $J$ Med. 2011;365(26):2484-2496. doi:10.1056/NEJMoa1103799

3. Burger RA, Brady MF, Bookman MA, et al. Incorporation of bevacizumab in the primary treatment of ovarian cancer. $N$ Engl $J$ Med. 2011;365(26):2473-2483. doi:10.1056/NEJMoa1104390

4. Jayson GC, Kohn EC, Kitchener HC, et al. Ovarian cancer. Lancet. 2014;384(9951):1376-1388. doi:10.1016/S0140-6736(13)62146-7

5. Parkes E, McCabe N, Kennedy R. Chapter 36 - Development of PARP inhibitors for BRCA-deficient epithelial ovarian cancer. In: Dammacco F, Silvestris F, editors. Oncogenomics: From Basic Research to Precision Medicine. London, UK: Academic Press; 2019:521-532.
6. Champia S, Ileana E, Giaccone G, et al. Incorporating immune-checkpoint inhibitors into systemic therapy of NSCLC. J Thorac Oncol. 2014;9:144-153. doi:10.1097/JTO.0000000000000074

7. Alme AK, Karir BS, Faltas BM, Drake CG. Blocking immune checkpoints in prostate, kidney, and urothelial cancer: an overview. Urol Oncol. 2016;34:171-181. doi:10.1016/j.urolonc.20 16.01.006

8. Schlienger K, Chu CS, Woo EY, et al. TRANCE-and CD40 ligand-matured dendritic cells reveal MHC class I-restricted $\mathrm{T}$ cells specific for autologous tumor in late-stage ovarian cancer patients. Clin Cancer Res. 2003;9:1517-1527.

9. Goodell V, Salazar LG, Urban N, et al. Antibody immunity to the p53 oncogenic protein is a prognostic indicator in ovarian cancer. J Clin Oncol. 2006;24:762-768. doi:10.1200/JCO.2005. 03.2813

10. Oda K, Hamanishi J, Matsuo K, Hasegawa K. Genomics to immunotherapy of ovarian clear cell carcinoma: unique opportunities for management. Gynecol Oncol. 2018;151:381-389. doi:10.1016/j. ygyno.2018.09.001

11. Longoria TC, Eskander RN. Immunotherapy in endometrial cancer - an evolving therapeutic paradigm. Gynecol Oncol Res Pract. 2015;2:11. doi:10.1186/s40661-015-0020-3

12. Zhang L, Conejo-Garcia JR, Katsaros D, et al. Intratumoral T cells, recurrence, and survival in epithelial ovarian cancer. $N$ Engl J Med. 2003;348(3):203-213. doi:10.1056/NEJMoa020177

13. Hamanishi J, Mandai M, Iwasaki M, et al. Programmed cell death 1 ligand 1 and tumor-infiltrating $\mathrm{CD} 8^{+} \mathrm{T}$ lymphocytes are prognostic factors of human ovarian cancer. Proc Natl Acad Sci U S A. 2007;104(9):3360-3365. doi:10.1073/pnas.0611533104

14. Lanitis E, Dangaj D, Irving M, Coukos G. Mechanisms regulating T cell infiltration and activity in solid tumors. Ann Oncol. 2017;28 (suppl_12):xii18-xii32. doi:10.1093/annonc/mdx238

15. Hegde PS, Karanikas V, Evers S. The where, the when, and the how of immune monitoring for cancer immunotherapies in the era of checkpoint inhibition. Clin Cancer Res. 2016;22(8):1865-1874. doi:10.1158/1078-0432.CCR-15-1507

16. Galon J, Bruni D. Approaches to treat immune hot, altered and cold tumors with combination immunotherapies. Nat Rev Drug Discov. 2019;18(3):197-218. doi:10.1038/s41573-018-0007-y

17. Curiel TJ, Coukos G, Zou L, et al. Specific recruitment of regulatory $\mathrm{T}$ cells in ovarian carcinoma fosters immune privilege and predicts reduced survival. Nat Med. 2004;10(9):942-949. doi:10.1038/nm1093

18. Gabrilovich DI, Nagaraj S. Myeloid-derived suppressor cells as regulators of the immune system. Nat Rev Immunol. 2009;9(3): 162-174. doi:10.1038/nri2506

19. Hodi FS, Mihm MC, Soiffer RJ, et al. Biologic activity of cytotoxic $\mathrm{T}$ lymphocyte-associated antigen 4 antibody blockade in previously vaccinated metastatic melanoma and ovarian carcinoma patients. Proc Natl Acad Sci U S A. 2003;100(8):4712-4717. doi:10.1073/ pnas.0830997100

20. Hodi FS, Butler M, Oble DA, et al. Immunologic and clinical effects of antibody blockade of cytotoxic T lymphocyte-associated antigen 4 in previously vaccinated cancer patients. Proc Natl Acad Sci U S A. 2008;105(8):3005-3010. doi:10.1073/pnas.0712237105

21. De Felice F, Marchetti C, Palaia I, et al. Immunotherapy of ovarian cancer: the role of checkpoint inhibitors. J Immunol Res. 2015;2015:191832. doi:10.1155/2015/191832

22. Hamanishi J, Mandai M, Ikeda T, et al. Safety and antitumor activity of anti-PD-1 antibody, nivolumab, in patients with platinum-resistant ovarian cancer. J Clin Oncol. 2015;33(34):40 15-4022. doi:10.1200/JCO.2015.62.3397

23. Disis ML, Taylor MH, Kelly K, et al. Efficacy and safety of avelumab for patients with recurrent or refractory ovarian cancer: phase $1 \mathrm{~b}$ results from the JAVELIN solid tumor trial. JAMA Oncol. 2019;5(3):393-401. doi:10.1001/jamaoncol.2018.6258 
24. Liu JF, Gordon M, Veneris J, et al. Safety, clinical activity and biomarker assessments of atezolizumab from a phase I study in advanced/recurrent ovarian and uterine cancers. Gynecol Oncol. 2019;154(2):314-322. doi:10.1016/j.ygyno.2019.05.021

25. Ghisoni E, Imbimbo M, Zimmermann S, Valabrega G. Ovarian cancer immunotherapy: turning up the heat. Int $J$ Mol Sci. 2019;20(12):2927. doi:10.3390/ijms20122927

26. Sabbatini P, Harter P, Scambia G, et al. Abagovomab as maintenance therapy in patients with epithelial ovarian cancer: a phase III trial of the AGO OVAR, COGI, GINECO, and GEICO--the MIMOSA study. J Clin Oncol. 2013;31(12):1554-1561. doi:10.1200/JCO.2012.46.4057

27. Buzzonetti A, Fossati M, Catzola V, Scambia G, Fattorossi A, Battaglia A. Immunological response induced by abagovomab as a maintenance therapy in patients with epithelial ovarian cancer: relationship with survival - a substudy of the MIMOSA trial. Cancer Immunol Immunother. 2014;63(10):1037-1045. doi:10.1007/s00262-014-1569-0

28. Battaglia A, Fossati M, Buzzonetti A, Scambia G, Fattorossi A. A robust immune system conditions the response to abagovomab (anti-idiotypic monoclonal antibody mimicking the CA125 protein) vaccination in ovarian cancer patients. Immunol Lett. 2017;191: 35-39. doi:10.1016/j.imlet.2017.09.006

29. Motz GT, Santoro SP, Wang LP, et al. Tumor endothelium FasL establishes a selective immune barrier promoting tolerance in tumors. Nat Med. 2014;20(6):607-615. doi:10.1038/nm.3541

30. Shrimali RK, Yu Z, Theoret MR, Chinnasamy D, Restifo NP, Rosenberg SA. Antiangiogenic agents can increase lymphocyte infiltration into tumor and enhance the effectiveness of adoptive immunotherapy of cancer. Cancer Res. 2010;70(15):6171-6180. doi:10.1158/0008-5472.CAN-10-0153

31. Li B, Lalani AS, Harding TC, et al. Vascular endothelial growth factor blockade reduces intratumoral regulatory $\mathrm{T}$ cells and enhances the efficacy of a GM-CSF-secreting cancer immunotherapy. Clin Cancer Res. 2006;12(22):6808-6816. doi:10.1158/1078-0432.CCR06- 1558

32. Lee JM, Cimino-Mathews A, Peer CJ, et al. Safety and clinical activity of the programmed death-ligand 1 inhibitor durvalumab in combination with poly (ADP- ribose) polymerase inhibitor olaparib or vascular endothelial growth factor receptor 1-3 inhibitor cediranib in women's cancers: a dose-escalation, phase I study. J Clin Oncol. 2017;35(19):2193-2202. doi:10.1200/JCO.2016.72.1340

33. Liu JF, Herold C, Gray KP, et al. Assessment of combined nivolumab and bevacizumab in relapsed ovarian cancer: a phase 2 clinical trial JAMA Oncol. 2019;5(12):1731. doi:10.1001/jamaoncol.2019.3343

34. Wang Z, Sun K, Xiao Y, et al. Niraparib activates interferon signaling and potentiates anti-PD-1 antibody efficacy in tumor models. Sci Rep. 2019;9(1):1853. doi:10.1038/s41598-019-38534-6

35. Ding L, Kim HJ, Wang Q, et al. PARP inhibition elicits STING-dependent antitumor immunity in Brcal-deficient ovarian cancer. Cell Rep. 2018;25 (11):2972-2980.e5. doi:10.1016/j.celrep.2018.11.054

36. Higuchi T, Flies DB, Marjon NA, et al. CTLA-4 blockade synergizes therapeutically with PARP inhibition in BRCA1-deficient ovarian cancer. Cancer Immunol Res. 2015;3(11):1257-1268. doi:10.1158/2326-6066.CIR-15-0044

37. Shen J, Zhao W, Ju Z, et al. PARPi triggers the STING-dependent immune response and enhances the therapeutic efficacy of immune checkpoint blockade independent of BRCAness. Cancer Res. 2019;79(2):311-319. doi:10.1158/0008-5472.CAN-18-1003

38. Drew Y, de Jonge $\mathrm{M}$, Hong $\mathrm{SH}$, et al. An open-label, phase II basket study of olaparib and durvalumab (MEDIOLA): results in germline BRCA -mutated (gBRCA m) platinum-sensitive relapsed (PSR) ovarian cancer (OC). Gynecol Oncol. 2018;149:246-247. doi:10.1016/j.ygyno.2018.04.555

39. Konstantinopoulos PA, Waggoner S, Vidal GA, et al. Single-arm phases 1 and 2 trial of niraparib in combination with pembrolizumab in patients with recurrent platinum-resistant ovarian carcinoma. JAMA Oncol. 2019;5(8):1141. doi:10.1001/jamaoncol.2019.1048
40. Tesaro, Inc. Niraparib in combination with pembrolizumab in patients with triple-negative breast cancer or ovarian cancer (TOPACIO). Available from: https://clinicaltrials.gov/ct2/show/ NCT02657889. NLM identifier: NCT02657889. Accessed January $30,2020$.

41. Zimmer AS, Nichols E, Cimino-Mathews A, et al. A phase I study of the PD-L1 inhibitor, durvalumab, in combination with a PARP inhibitor, olaparib, and a VEGFR1-3 inhibitor, cediranib, in recurrent women's cancers with biomarker analyses. J Immunother Cancer. 2019;7(1):197.

42. National Cancer Institute (NCI). Phase I/II study of anti-programmed death ligand-1 antibody MEDI4736 in combination with olaparib and/or cediranib for advanced solid tumors and advanced or recurrent ovarian, triple negative breast, lung, prostate and colorectal cancers. Available from: https://clinicaltrials.gov/ct2/ show/NCT02484404. NLM identifer: NCT02484404. Accessed January 30, 2020.

43. National comprehensive cancer network guidelines. Ovarian cancer including fallopian tube cancer and primary peritoneal cancer (Version 3.2019 - November 26, 2019). Available from: https:// www.nccn.org/professionals/physician_gls/pdf/ovarian.pdf.

44. Lazzari C, Karachaliou N, Bulotta A, et al. Combination of immunotherapy with chemotherapy and radiotherapy in lung cancer: is this the beginning of the end for cancer? Ther Adv Med Oncol. 2018;10:1758835918762094. doi:10.1177/1758835918762094

45. Pfizer. Avelumab in previously untreated patients with epithelial ovarian cancer (JAVELIN OVARIAN 100). Available from: https:// clinicaltrials.gov/ct2/show/NCT02718417. NLM identifier: NCT02 718417. Accessed January 30, 2020.

46. Brewer M, Angioli R, Scambia G, et al. Front-line chemo-immunotherapy with carboplatin-paclitaxel using oregovomab indirect immunization in advanced ovarian cancer: a randomized phase II study. Gynecol Oncol. 2020;156(3):52 3-529. doi:10.1016/j.ygyno.2019.12.024

47. Battaglia A, Buzzonetti A, Fossati M, et al. Translational immune correlates of indirect antibody immunization in a randomized phase II study using scheduled combination therapy with carboplatin/ paclitaxel plus oregovomab in ovarian cancer patients. Cancer Immunol Immunother. 2020;69(3):383-397. doi:10.1007/s00262019-02456-z

48. Kandalaft LE, Odunsi K, Coukos G. Immunotherapy in ovarian cancer: are we there yet? J Clin Oncol. 2019;37(27):2460-2471. doi: $10.1200 /$ JCO.19.00508

49. Zhang Z, Yu X, Wang Z, Wu P, Huang J. Anthracyclines potentiate anti-tumor immunity: a new opportunity for chemoimmunotherapy. Cancer Lett. 2015;369(2):331-335. doi:10.1016/j.canlet.2015.10. 002

50. O'Cearbhaill RE, Wolfer A, Disilvestro P, et al. 945PA phase I/II study of chemo-immunotherapy with durvalumab (durva) and pegylated liposomal doxorubicin (PLD) in platinum-resistant recurrent ovarian cancer (PROC). Ann Oncol. 2018;29(suppl 8):viii337. doi:10.1093/annonc/mdy285.153

51. Matulonis U, Barry W, Penson R, et al. Phase II study of pembrolizumab (pembro) combined with pegylated liposomal doxorubicin (PLD) for recurrent platinum-resistant ovarian, fallopian tube or peritoneal cancer. SGO Annual Meeting. 2018;149:24-27.

52. Monk BJ, Brady MF, Aghajanian C, et al. A phase 2, randomized, double-blind, placebo- controlled study of chemo-immunotherapy combination using motolimod with pegylated liposomal doxorubicin in recurrent or persistent ovarian cancer: a gynecologic oncology group partners study. Ann Oncol. 2017;28(5):996-1004. doi:10.1093/annonc/mdx049

53. Pujade-Lauraine E, Fujiwara K, Dychter SS, Devgan G, Monk BJ. Avelumab (anti-PD-L1) in platinum-resistant/refractory ovarian cancer: JAVELIN ovarian 200 phase III study design. Future Oncol. 2018;14(21):2103-2113. doi:10.2217/fon-2018-0070 
54. Madondo MT, Quinn M, Plebanski M. Low dose cyclophosphamide: mechanisms of $\mathrm{T}$ cell modulation. Cancer Treat Rev. 2016;42:3-9. doi:10.1016/j.ctrv.2015.11.005

55. Mkrtichyan M, Najjar YG, Raulfs EC, et al. Anti-PD-1 synergizes with cyclophosphamide to induce potent anti-tumor vaccine effects through novel mechanisms. Eur J Immunol. 2011;41(10):29 77-2986. doi:10.1002/eji.201141639

56. Roswell Park Cancer Institute. Pembrolizumab, bevacizumab, and cyclophosphamide in treating patients with recurrent ovarian, fallopian tube, or primary peritoneal cancer. Available from: https:// clinicaltrials.gov/ct2/show/NCT02853318. NLM identifier: NCT02 853318. Accessed January 30, 2020.

57. Zsiros E, Frederick PJ, Akers SN, et al. A phase II trial of pembrolizumab in combination with bevacizumab and oral metronomic cyclophosphamide for recurrent epithelial ovarian, fallopian tube or primary peritoneal cancer. Gynecol Oncol. 2019;154(suppl.1, 23):2-288. doi:10.1016/j.ygyno.2019.04.056

58. Matulonis UA, Moore KN, Martin LP, et al. Initial safety and activity findings from a phase IB escalation study of mirvetuximab soravtansine, a folate receptor alpha (FR $\alpha$-targeting antibody-drug conjugate (ADC), with pembrolizumab in platinum-resistant epithelial ovarian cancer (EOC) patients. Gynecol Oncol. 2018; 149:38. doi:10.1016/j.ygyno.2018.04.085

59. ImmunoGen, Inc. Study of mirvetuximab soravtansine in comb. with bevacizumab, carboplatin, PLD, pembrolizumab, or bevacizumab +carboplatin in adults with Fra+Adv. EOC, primary peritoneal or fallopian tube cancer. Available from: https://clinicaltrials.gov/ct2/ show/NCT02606305. NLM identifier: NCT02606305. Accessed January 30, 2020.

60. Wolchok JD, Kluger $\mathrm{H}$, Callahan $\mathrm{MK}$, et al. Nivolumab plus ipilimumab in advanced melanoma. $N$ Engl $\mathrm{J} \mathrm{Med.} \mathrm{2013;369}$ (2):122-133. doi:10.1056/NEJMoa1302369

61. Burger RA, Sill MW, Zamarin D, et al. NRG oncology phase II randomized trial of nivolumab with or without ipilimumab with persistent or recurrent ovarian cancer [abstract]. Poster presented at: Biennial Meeting of the International Gynecologic Cancer Society (IGCS); September 14-16; 2018; Kyoto, Japan.

62. National Cancer Institute (NCI). Nivolumab with or without ipilimumab in treating patients with persistent or recurrent epithelial ovarian, primary peritoneal, or fallopian tube cancer. Available from: https://clinicaltrials.gov/ct2/show/NCT02498600. NLM identifier: NCT02498600. Accessed January 30, 2020.

63. Sanborn RE, Pishvaian MJ, Kluger HM, et al. Clinical results with combination of anti-CD27 agonist antibody, varlilumab, with anti-PD1 antibody nivolumab in advanced cancer patients. J Clin Oncol. 2017;35 (15_suppl):3007. doi:10.1200/JCO.2017.35.15_suppl.3007

64. Celldex Therapeutics. A dose escalation and cohort expansion study of anti-CD27 (Varlilumab) and anti-PD-1 (Nivolumab) in advanced refractory solid tumors. Available from: https://clinical trials.gov/ct2/show/NCT02335918. NLM identifier: NCT02335 918. Accessed January 30, 2020.

65. Perica K, Varela JC, Oelke M, Schneck J. Adoptive T cell immunotherapy for cancer. Rambam Maimonides Med J. 2015;6(1): e0004. doi:10.5041/RMMJ.10179

66. Carlsten M, Bjorkstrom NK, Norell H, et al. DNAX accessory molecule-1 mediated recognition of freshly isolated ovarian carcinoma by resting natural killer cells. Cancer Res. 2007;67 (3):1317-1325. doi:10.1158/0008-5472.CAN-06-2264

67. Hermanson DL, Bendzick L, Kaufman DS. Mouse xenograft model for intraperitoneal administration of NK cell immunotherapy for ovarian cancer. Methods Mol Biol. 2016;1441:277-284.

68. Liu J, Li H, Cao S, et al. Maintenance therapy with autologous cytokine-induced killer cells in patients with advanced epithelial ovarian cancer after first-line treatment. J Immunother. 2014;37 (2):115-122. doi:10.1097/CJI.0000000000000021
69. Zhou Y, Chen CL, Jiang SW, et al. Retrospective analysis of the efficacy of adjuvant CIK cell therapy in epithelial ovarian cancer patients who received postoperative chemotherapy. Oncoimmunology. 2019;8(2): e1528411. doi:10.1080/2162402X.2018.1528411

70. Aoki Y, Takakuwa K, Kodama S, et al. Use of adoptive transfer of tumor-infiltrating lymphocytes alone or in combination with cisplatin-containing chemotherapy in patients with epithelial ovarian cancer. Cancer Res. 1991;51(7):1934-1939.

71. Ikarashi H, Fujita K, Takakuwa K, et al. Immunomodulation in patients with epithelial ovarian cancer after adoptive transfer of tumor-infiltrating lymphocytes. Cancer Res. 1994;54(1):190-196.

72. Freedman RS, Edwards CL, Kavanagh JJ, et al. Intraperitoneal adoptive immunotherapy of ovarian carcinoma with tumor-infiltrating lymphocytes and low-dose recombinant interleukin-2: a pilot trial. $J$ Immunother Emphasis Tumor Immunol. 1994;16(3):198-210. doi:10.1097/00002371-199410000-00004

73. Fujita K, Ikarashi H, Takakuwa K, et al. Prolonged disease-free period in patients with advanced epithelial ovarian cancer after adoptive transfer of tumor-infiltrating lymphocytes. Clin Cancer Res. 1995;1(5):501-507.

74. Pedersen M, Westergaard MCW, Milne K, et al. Adoptive cell therapy with tumor-infiltrating lymphocytes in patients with metastatic ovarian cancer: a pilot study. Oncoimmunology. 2018;7(12): e1502905. doi:10.1080/2162402X.2018.1502905

75. Westergaard MCW, Andersen R, Chong C, et al. Tumor-reactive $\mathrm{T}$ cell subsets in the microenvironment of ovarian cancer. $\mathrm{Br}$ $J$ Cancer. 2019;120(4):424-434. doi:10.1038/s41416-019-0384-y

76. Felder M, Kapur A, Gonzalez-Bosquet J, et al. MUC16 (CA125): tumor biomarker to cancer therapy, a work in progress. Mol Cancer. 2014;13:129. doi:10.1186/1476-4598-13-129

77. Zhu X, Cai H, Zhao L, Ning L, Lang J. CAR-T cell therapy in ovarian cancer: from the bench to the bedside. Oncotarget. 2017;8 (38):64607-64621. doi:10.18632/oncotarget.19929

78. Hassan R, Kreitman RJ, Pastan I, Willingham MC. Localization of mesothelin in epithelial ovarian cancer. Appl Immunohistochem Mol Morphol. 2005;13(3):243-247. doi:10.1097/01.pai.00000141545.364 85.d6

79. Napoletano C, Bellati F, Tarquini E, et al. MAGE-A and NY-ESO1 expression in cervical cancer: prognostic factors and effects of chemotherapy. Am J Obstet Gynecol. 2008;198(1):99-e1. doi:10. 1016/j.ajog.2007.05.019

80. Bellati F, Napoletano C, Tarquini E, et al. Cancer testis antigen expression in primary and recurrent vulvar cancer: association with prognostic factors. Eur J Cancer. 2007;43(17):2621-2627. doi:10. 1016/j.ejca.2007.08.031

81. Chitale DA, Jungbluth AA, Marshall DS, et al. Expression of cancer-testis antigens in endometrial carcinomas using a tissue microarray. Mod Pathol. 2005;18(1):119-126. doi:10.1038/modpathol. 3800232

82. Odunsi K, Jungbluth AA, Stockert E, et al. NY-ESO-1 and LAGE-1 cancer-testis antigens are potential targets for immunotherapy in epithelial ovarian cancer. Cancer Res. 2003;63(18):6076-6083.

83. Chekmasova AA, Rao TD, Nikhamin Y, et al. Successful eradication of established peritoneal ovarian tumors in SCID-Beige mice following adoptive transfer of $\mathrm{T}$ cells genetically targeted to the MUC16 antigen. Clin Cancer Res. 2010;16(14):3594-3606. doi:10.1158/1078-0432.CCR-10-0192

84. Carpenito C, Milone MC, Hassan R, et al. Control of large, established tumor xenografts with genetically retargeted human $\mathrm{T}$ cells containing CD28 and CD137 domains. Proc Natl Acad Sci USA. 2009;106:3360-3365. doi:10.1073/pnas.0813101106

85. Tanyi JL, Haas AR, Beatty GL, et al. Anti-mesothelin chimeric antigen receptor $\mathrm{T}$ cells in patients with epithelial ovarian cancer. $J$ Clin Oncol. 2016;34(15_suppl):5511. doi:10.1200/JCO.2016. 34.15_supp1.5511 
86. Song DG, Ye Q, Carpenito C, et al. In vivo persistence, tumor localization, and antitumor activity of CAR-engineered $\mathrm{T}$ cells is enhanced by costimulatory signaling through CD137 (4-1BB). Cancer Res. 2011;71(13):4617-4627. doi:10.1158/0008-5472. CAN-11-0422

87. Anderson KG, Voillet V, Bates BM, et al. Engineered adoptive T-cell therapy prolongs survival in a preclinical model of advanced-stage ovarian cancer. Cancer Immunol Res. 2019;7 (9):1412-1425. doi:10.1158/2326-6066.CIR-19-0258

88. Marth C, Wieser V, Tsibulak I, et al. Immunotherapy in ovarian cancer: fake news or the real deal? Int J Gynecol Cancer. 2019;29 (1):201-211. doi:10.1136/ijgc-2018-000011

89. Odunsi K, Matsuzaki J, Karbach J, et al. Efficacy of vaccination with recombinant vaccinia and fowlpox vectors expressing NY-ESO-1 antigen in ovarian cancer and melanoma patients. Proc Natl Acad Sci U S A. 2012;109(15):5797-5802. doi:10.10 73/pnas. 1117208109

90. Odunsi K, Matsuzaki J, James SR, et al. Epigenetic potentiation of NY-ESO-1 vaccine therapy in human ovarian cancer. Cancer Immunol Res. 2014;2(1):37-49. doi:10.1158/2326-6066.CIR-130126

91. Sabbatini P, Tsuji T, Ferran L, et al. Phase I trial of overlapping long peptides from a tumor self-antigen and poly-ICLC shows rapid induction of integrated immune response in ovarian cancer patients. Clin Cancer Res. 2012;18(23):6497-6508. doi:10.1158/ 1078-0432.CCR-12-2189

92. Tsuji T, Sabbatini P, Jungbluth AA, et al. Effect of Montanide and poly-ICLC adjuvant on human self/tumor antigen-specific CD4+ $\mathrm{T}$ cells in phase I overlapping long peptide vaccine trial. Cancer Immunol Res. 2013;1(5):340-350. doi:10.1158/2326-6066.CIR-130089

93. Szender JB, Papanicolau-Sengos A, Eng KH, et al. NY-ESO-1 expression predicts an aggressive phenotype of ovarian cancer Gynecol Oncol. 2017;145(3):420-425. doi:10.1016/j.ygyno.2017. 03.509

94. Xu Z, Tang H, Zhang T, et al. TEX19 promotes ovarian carcinoma progression and is a potential target for epitope vaccine immunotherapy. Life Sci. 2020;241:117171. doi:10.1016/j.lfs.2019. 117171

95. Napoletano C, Rughetti A, Landi R, et al. Immunogenicity of allo-vesicle carrying ERBB2 tumor antigen for dendritic cell-based anti-tumor immunotherapy. Int J Immunopathol Pharmacol. 2009;22(3):647-658. doi: $10.1177 / 039463200902200310$

96. Napoletano C, Pinto D, Bellati F, et al. A comparative analysis of serum and serum-free media for generation of clinical grade DCs. J Immunother. 2007;30(5):567-576. doi:10.1097/CJI.0b013e318046f396

97. Gray HJ, Benigno B, Berek J, et al. Progression-free and overall survival in ovarian cancer patients treated with CVac, a mucin 1 dendritic cell therapy in a randomized phase 2 trial. J Immunother Cancer. 2016;4:34. doi:10.1186/s40425-016-0137-x

98. Lawrence MS, Stojanov P, Polak P, et al. Mutational heterogeneity in cancer and the search for new cancer-associated genes. Nature. 2013;499(7457):214-218. doi:10.1038/nature12213

99. Sarivalasis A, Boudousquié C, Balint K, et al. A Phase I/II trial comparing autologous dendritic cell vaccine pulsed either with personalized peptides (PEP-DC) or with tumor lysate (OC-DC) in patients with advanced high-grade ovarian serous carcinoma. J Transl Med. 2019;17(1):391. doi:10.1186/s12967-019-02133-w

100. Odunsi K, Qian F, Matsuzaki J, et al. Vaccination with an NY-ESO1 peptide of HLA class I/II specificities induces integrated humoral and T cell responses in ovarian cancer. Proc Natl Acad Sci U S A. 2007;104(31):12837-12842. doi:10.1073/pnas.0703342104

101. Pardoll DM. The blockade of immune checkpoints in cancer immunotherapy. Nat Rev Cancer. 2012;12(4):252-264. doi:10.10 $38 / \mathrm{nrc} 3239$
102. Heong V, Ngoi N, Tan DS. Update on immune checkpoint inhibitors in gynecological cancers. J Gynecol Oncol. 2017;28(2):e20. doi:10.3802/jgo.2017.28.e20

103. Chan JJ, Tan SH, Lim TW, et al. Oregovomab (orego) and nivolumab (nivo) as a combinatorial immunotherapy strategy for recurrent epithelial ovarian cancer (rEOC): ORION-01 phase Ib. Ann Oncol. 2018;29(suppl_8):mdy285.186. doi:10.1093/annonc/mdy285.186

104. National Cancer Centre, Singapore. Phase Ib/IIa trial to evaluate oregovomab and nivolumab in epithelial cancer of ovarian, tubal or peritoneal origin (ORION-01). Available from: https://clinicaltrials. gov/ct2/show/NCT03100006. NLM identifier: NCT03100006. Accessed January 30, 2020.

105. Hoffmann-La Roche. A Study of Atezolizumab Versus Placebo in Combination With Paclitaxel, Carboplatin, and Bevacizumab in Participants With Newly-Diagnosed Stage III or Stage IV Ovarian, Fallopian Tube, or Primary Peritoneal Cancer (IMagyn050). Available from: https://clinicaltrials.gov/ct2/show/ NCT03038100. NLM identifier: NCT03038100. Accessed January 30, 2020.

106. ARCAGY/ GINECO GROUP. ATALANTE: Atezolizumab vs Placebo Phase III Study in Late Relapse Ovarian Cancer Treated With Chemotherapy+Bevacizumab (ATALANTE). Available from: https://clinicaltrials.gov/ct2/show/NCT02891824. NLM identifier: NCT02891824. Accessed January 30, 2020.

107. European Organisation for Research and Treatment of Cancer EORTC. Anti-programmed Cell Death-1 Ligand 1 (aPDL-1) Antibody Atezolizumab, Bevacizumab and Acetylsalicylic Acid in Recurrent Platinum Resistant Ovarian Cancer. Available from: https://clinicaltrials.gov/ct2/show/NCT02659384. NLM identifier: NCT02659384. Accessed January 30, 2020.

108. National Cancer Institute (NCI). Pegylated Liposomal Doxorubicin Hydrochloride With Atezolizumab and/or Bevacizumab in Treating Patients With Recurrent Ovarian, Fallopian Tube, or Primary Peritoneal Cancer. Available from: https://clinicaltrials.gov/ct2/show/ NCT02839707. NLM identifier: NCT02839707. Accessed January 30, 2020.

109. Grupo Español de Investigación en Cáncer de Ovario. Platinumbased Chemotherapy With Atezolizumab and Niraparib in Patients With Recurrent Ovarian Cancer (ANITA). Available from: https:// clinicaltrials.gov/ct2/show/NCT03598270. NLM identifier: NCT03598270. Accessed January 30, 2020.

110. AGO Research GmbH. Atezolizumab With Bevacizumab and Chemotherapy vs Bevacizumab and Chemotherapy in Early Relapse Ovarian Cancer. Available from: https://clinicaltrials.gov/ct2/show/ NCT03353831. NLM identifier: NCT03353831. Accessed January 30, 2020.

111. AstraZeneca. Durvalumab Treatment in Combination With Chemotherapy and Bevacizumab, Followed by Maintenance Durvalumab, Bevacizumab and Olaparib Treatment in Advanced Ovarian Cancer Patients. (DUO-O). Available from: https://clinical trials.gov/ct2/show/NCT03737643. NLM identifier: NCT037 37643. Accessed January 30, 2020.

112. Ludwig Institute for Cancer Research. Phase $1 / 2$ Study of Motolimod, Doxorubicin, and Durvalumab in Recurrent, Platinum-Resistant Ovarian Cancer. Available from: https:/clinical trials.gov/ct2/show/NCT02431559. NLM identifier: NCT0243 1559. Accessed January 30, 2020.

113. M.D. Anderson Cancer Center. Matched Paired Pharmacodynamics and Feasibility Study of Durvalumab in Combination With Chemotherapy in Frontline Ovarian Cancer (N-Dur). Available from: https://clinicaltrials.gov/ct2/show/NCT02726997. NLM identifier: NCT02726997. Accessed January 30, 2020.

114. Dana-Farber Cancer Institute. A Phase II Study Of Nivolumab/ Bevacizumab/Rucaparib. Available from: https://clinicaltrials.gov/ ct2/show/NCT02873962. NLM identifier: NCT02873962. Accessed January 30, 2020. 
115. Clovis Oncology, Inc. A Study in Ovarian Cancer Patients Evaluating Rucaparib and Nivolumab as Maintenance Treatment Following Response to Front-Line Platinum-Based Chemotherapy (ATHENA). Available from: https://clinicaltrials.gov/ct2/show/ NCT03522246. NLM identifier: NCT03522246. Accessed January 30, 2020.

116. New Mexico Cancer Care Alliance. PARP-inhibition and CTLA-4 Blockade in BRCA-deficient Ovarian Cancer. Available from: https:/clinicaltrials.gov/ct2/show/NCT02571725. NLM identifier: NCT02571725. Accessed January 30, 2020.

117. Sidney Kimmel Comprehensive Cancer Center at Johns Hopkins. Study of Tremelimumab Alone or Combined With Olaparib for Patients With Persistent EOC (Epithelial Ovarian, Fallopian Tube or Primary Peritoneal Carcinoma). Available from: https://clinicaltrials.gov/ct2/ show/NCT02485990. NLM identifier: NCT02485990. Accessed January 30, 2020.

118. Pfizer. Avelumab and Talazoparib in Untreated Advanced Ovarian Cancer (JAVELIN OVARIAN PARP 100). Available from: https:// clinicaltrials.gov/ct2/show/NCT03642132. NLM identifier: NCT03642132. Accessed January 30, 2020.

119. Pfizer. A Study Of Avelumab Alone Or In Combination With Pegylated Liposomal Doxorubicin Versus Pegylated Liposomal Doxorubicin Alone In Patients With Platinum Resistant/ Refractory Ovarian Cancer (JAVELIN Ovarian 200). Available from: https://clinicaltrials.gov/ct2/show/NCT02580058. NLM identifier: NCT02580058. Accessed January 30, 2020.

120. Merck Sharp \& Dohme Corp. Study of Chemotherapy With Pembrolizumab (MK-3475) Followed by Maintenance With Olaparib (MK-7339) for the First-Line Treatment of Women With BRCA Non-mutated Advanced Epithelial Ovarian Cancer (EOC) (MK-7339-001/KEYLYNK-001/ENGOT-ov43). Available from: https://clinicaltrials.gov/ct2/show/NCT03740165. NLM identifier: NCT03740165. Accessed January 30, 2020.

121. H. Lee Moffitt Cancer Center and Research Institute. Dose Dense Paclitaxel With Pembrolizumab (MK-3475) in Platinum Resistant Ovarian Cancer. Available from: https://clinicaltrials.gov/ct2/show/NCT02440425. NLM identifier: NCT02440425. Accessed January 30, 2020.

122. M.D. Anderson Cancer Center. Pembrolizumab, Carboplatin, and Paclitaxel in Treating Patients With Stage III-IV Ovarian, Primary Peritoneal, or Fallopian Tube Cancer. Available from: https://clin icaltrials.gov/ct2/show/NCT02520154. NLM identifier: NCT02520154. Accessed January 30, 2020.

123. Medical College of Wisconsin. Phase II: Pembrolizumab/ Carboplatin/Taxol in Epithelial Ovary Cancer. Available from: https://clinicaltrials.gov/ct2/show/NCT02766582. NLM identifier: NCT02766582. Accessed January 30, 2020.

124. Tesaro, Inc. A Phase 3 Comparison of Platinum-based Therapy With TSR-042 and Niraparib Versus Standard of Care (SOC) Platinum-based Therapy as First-line Treatment of Stage III or IV Nonmucinous Epithelial Ovarian Cancer (FIRST). Available from: https://clinicaltrials.gov/ct2/show/NCT03602859. NLM identifier: NCT03602859. Accessed January 30, 2020.

125. Nordic Society for Gynaecologic Oncology. Trial Comparing Niraparib-bevacizumab-TSR042 and Niraparib-bevacizumab to Standard of Care in Recurrent Ovarian Cancer. Available from: https://clinicaltrials.gov/ct2/show/NCT03806049. NLM identifier: NCT03806049. Accessed January 30, 2020.

126. The First People's Hospital of Changzhou. Radiofrequency Ablation Combined With Cytokine-induced Killer Cells for the Patients With Ovarian Carcinoma. Available from: https://clinical trials.gov/ct2/show/NCT02487693. NLM identifier: NCT024 87693. Accessed January 30, 2020.

127. Shanghai 6th People's Hospital. The Clinical Research of Fourth Generation CART-cell Therapy in Refractory-Relapsed Ovarian Cancer. Available from: https://clinicaltrials.gov/ct2/show/NCT03814447. NLM Identifier: NCT03814447. Accessed January 30,2020.
128. Allife Medical Science and Technology Co., Ltd. Clinical Study on the Safety and Efficacy of Anti-Mesothelin Car NK Cells With Epithelial Ovarian Cancer. Available from: https://clinicaltrials. gov/ct2/show/NCT03692637. NLM Identifier: NCT03692637. Accessed January 30, 2020.

129. University of Pennsylvania. Phase I Clinical Trial of Adoptive Transfer of Autologous Folate Receptor - Alpha Redirected T Cells for Recurrent High Grade Serous Ovarian, Fallopian Tube, or Primary Peritoneal Cancer. Available from: https://clinicaltrials.gov/ct2/show/NCT03585764. NLM Identifier: NCT03585764. Accessed January 30, 2020.

130. Chinese PLA General Hospital. Clinical Study of Chimeric HER-2 Antigen Receptor-modified T Cells in Chemotherapy Refractory HER-2 Advanced Solid Tumors. Available from: https://clinicaltrials.gov/ct2/show/ NCT01935843. NLM Identifier: NCT01935843. Accessed January 30, 2020.

131. Chinese PLA General Hospital. Clinical Study of Chimeric CD (Cluster of Differentiation)133 Antigen Receptor-modified T Cells in Relapsed and/or Chemotherapy Refractory Malignancies. Available from: https://clinicaltrials.gov/ct2/show/NCT02541370. NLM Identifier: NCT02541370. Accessed January 30, 2020.

132. Adaptimmune. A Phase I/IIa, Open Label Clinical Trial Evaluating the Safety and Efficacy of Autologous $\mathrm{T}$ Cells Expressing Enhanced TCRs Specific for NY-ESO-1 in Patients With Recurrent or Treatment Refractory Ovarian Cancer. Available from: https://clinicaltrials.gov/ct2/show/NCT01567891. NLM Identifier: NCT01567891. Accessed January 30, 2020.

133. National Cancer Institute (NCI). A Phase I, Open Label Study Evaluating the Safety and Efficacy of Adoptive Transfer of Autologous NY-ESO-1 CD8-TCR Engineered T Cells and NYESO-1 CD4-TCR Engineered Hematopoietic Stem Cells (HSC) After a Myeloablative Conditioning Regimen, With Administration of IL-2 in Patients With Recurrent or Treatment Refractory Ovarian, Fallopian Tube or Primary Peritoneal Cancer. Available from: https://clinicaltrials.gov/ct2/show/NCT03691376. NLM Identifier: NCT03691376. Accessed January 30, 2020.

134. Shenzhen Second People's Hospital. Phase I Study of Malignancies That Express NY-ESO-1 With T Cell Receptor-transduced T Cells Targeting NYESO-1. Available from: https://clinicaltrials.gov/ct2/show/NCT02457650. NLM Identifier: NCT02457650. Accessed January 30, 2020.

135. University Health Network, Toronto. Phase Ib Study of TBI-1301 (NY-ESO1 Specific TCR Gene Transduced Autologous T Lymphocytes) in Patients With Solid Tumors. Available from: https://clinicaltrials.gov/ct2/show/ NCT02869217. NLM Identifier: NCT02869217. Accessed January 30, 2020.

136. Zhujiang Hospital. Application of NY-ESO-1-specific TCR Affinity Enhancing Specific T Cell Therapy (TAEST16001) in Solid Tumors Except Non Small Cell Lung Cancer,Including Liver Cancer,Gastric Cancer,Esophageal Cancer and so on. Available from: https://clinicaltrials.gov/ct2/show/NCT03159585. NLM Identifier: NCT03159585. Accessed January 30, 2020.

137. National Cancer Institute (NCI). A Phase I Open Label Clinical Trial Evaluating the Safety and Efficacy of Adoptive Transfer of NY-ESO-1 TCR Engineered Autologous T Cells in Combination With Decitabine in Patients With Recurrent or Treatment Refractory Ovarian Cancer. Available from: https://clinicaltrials.gov/ct2/show/NCT03017131. NLM Identifier: NCT03017131. Accessed January 30, 2020.

138. Memorial Sloan Kettering Cancer Center. A Phase I Clinical Trial of Cyclophosphamide Followed by Intravenous and Intraperitoneal Infusion of Autologous T Cells Genetically Engineered to Secrete IL-12 and to Target the MUC16ecto Antigen in Patients With Recurrent MUC16ecto+ Solid Tumors. Available from: https://clinicaltrials.gov/ct2/show/NCT02498912. NLM Identifier: NCT02498912. Accessed January 30, 2020.

139. Adaptimmune. Phase 1 Dose Escalation, Multi-tumor Study to Assess the Safety, Tolerability and Antitumor Activity of Genetically Engineered MAGE-A44 ${ }^{\mathrm{c} 1032} \mathrm{~T}$ in HLA-A2+ Subjects With MAGE-A4 Positive Tumors. Available from: https://clinicaltrials.gov/ct2/show/ NCT03132922. NLM Identifier: NCT03412877. Accessed January 30, 2020. 
140. National Cancer Institute (NCI). Administration of Autologous TCells Genetically Engineered to Express T-Cell Receptors Reactive Against Mutated Neoantigens in People With Metastatic Cancer. Available from: https://clinicaltrials.gov/ct2/show/NCT03412877. NLM Identifier: NCT03412877. Accessed January 30, 2020.

141. Memorial Sloan Kettering Cancer Center. A Phase I Study of Concomitant WT1 Analog Peptide Vaccine With Montanide and GM-CSF in Combination With Nivolumab in Patients With Recurrent Ovarian Cancer Who Are in Second or Greater Remission. Available from: https://clinicaltrials.gov/ct2/show/ NCT02737787. NLM Identifier: NCT02737787. Accessed January 30, 2020.

142. AstraZeneca. A Phase II Trial of TPIV200/huFR-1 (A MultiEpitope Anti-Folate Receptor Vaccine) Plus Anti-PD-L1 MEDI4736 (Durvalumab) in Patients With Platinum Resistant Ovarian Cancer. Available from: https://clinicaltrials.gov/ct2/ show/NCT02764333. NLM Identifier: NCT02764333. Accessed January 30, 2020.

143. University of Pennsylvania. Phase I Study of Human Chimeric Antigen Receptor Modified T Cells in Patients With Mesothelin Expressing Cancers. Available from: https://clinicaltrials.gov/ct2/ show/NCT03054298. NLM Identifier: NCT03054298. Accessed January 30, 2020.

144. Celyad. A Dose Escalation Phase I Study to Assess the Safety and Clinical Activity of Multiple Cancer Indications (THINK). Available from: https://clinicaltrials.gov/ct2/show/NCT03018405. NLM Identifier: NCT03054298. Accessed January 30, 2020.
145. Mie University. Multi-center, Investigator Initiated Phase 1 Study of NY-ESO-1 Specific TCR Gene Transferred T Lymphocytes With Solid Tumors. Available from: https://clinicaltrials.gov/ct2/show/ NCT02366546. NLM Identifier: NCT02366546. Accessed January 30, 2020.

146. Memorial Sloan Kettering Cancer Center. A Phase I Dose Escalation Safety and Feasibility Study of WT1-Specific T Cells for the Treatment of Patients With Advanced Ovarian, Primary Peritoneal, and Fallopian Tube Carcinomas. Available from: https://clinicaltrials.gov/ct2/show/NCT00562640. NLM Identifier: NCT02737787. Accessed January 30, 2020.

147. Chiou VL, Burotto M. Pseudoprogression and immune-related response in solid tumors. J Clin Oncol. 2015;33(31):3541-3543. doi:10.1200/JCO.2015.61.6870

148. Persigehl T, Lennartz S, Schwartz LH. iRECIST: how to do it. Cancer Imaging. 2020;20(1):2. doi:10.1186/s40644-019-0 281-x

149. Seymour L, Bogaerts J, Perrone A, et al. iRECIST: guidelines for response criteria for use in trials testing immunotherapeutics. Lancet Oncol. 2017;18(3):e143-e152. doi:10.1016/S1470-204 5(17)30074-8

\section{Publish your work in this journal}

OncoTargets and Therapy is an international, peer-reviewed, open access journal focusing on the pathological basis of all cancers, potential targets for therapy and treatment protocols employed to improve the management of cancer patients. The journal also focuses on the impact of management programs and new therapeutic agents and protocols on patient perspectives such as quality of life, adherence and satisfaction. The manuscript management system is completely online and includes a very quick and fair peer-review system, which is all easy to use. Visit http://www.dovepress.com/ testimonials.php to read real quotes from published authors. 